\title{
ZYGMUND VECTOR FIELDS, HILBERT TRANSFORM AND FOURIER COEFFICIENTS IN SHEAR COORDINATES
}

\author{
DRAGOMIR ŠARIĆ
}

\begin{abstract}
We parametrize the space $\mathcal{Z}$ of Zygmund vector fields on the unit circle in terms of infinitesimal shear functions on the Farey tesselation. Then we express the Hilbert transform and the Fourier coefficients of the Zygmund vector fields in terms of the above parametrization by infinitesimal shear functions. Finally, we compute the Weil-Petersson metric on the Teichmüller space of a punctured surface in terms of shears.
\end{abstract}

\section{INTRODUCTION}

The hyperbolic plane $\mathbf{H}$ has the unit circle $S^{1}$ as its ideal boundary. Vector fields on the unit circle $S^{1}$ are describing infinitesimal deformations of the circle maps. We study Zygmund vector fields which arise as tangent vectors to the space of quasisymmetric maps of $S^{1}$ at the identity map $i d: S^{1} \rightarrow S^{1}$ (see [15, 25]). The Teichmüller space $T(\mathbf{H})$ of the hyperbolic plane $\mathbf{H}$, called the universal Teichmüller space, consists of all quasisymmetric maps of the unit circle $S^{1}$ which fix $1, i,-1 \in$ $S^{1}$. Therefore, the space $\mathcal{Z}$ of all Zygmund vector fields on $S^{1}$ which vanish at $1, i,-1 \in S^{1}$ is the tangent space to the universal Teichmüller space $T(\mathbf{H})$ at the basepoint $i d \in T(\mathbf{H})$ (see [1]).

The Farey tesselation $\mathcal{F}$ of the hyperbolic plane $\mathbf{H}$ is a locally finite ideal triangulation of $\mathbf{H}$ which is invariant under the group of isometries of $\mathbf{H}$ generated by hyperbolic reflections in the sides of a complementary triangle of $\mathcal{F}$. The group of orientation preserving isometries of $\mathbf{H}$ that setwise preserve $\mathcal{F}$ is isomorphic to $P S L_{2}(\mathbf{Z})$ and it acts simply transitively on the oriented edges of $\mathcal{F}$. A vector field on $S^{1}$ naturally induces a real-valued function on the edges of $\mathcal{F}$, called the infinitesimal shear function of $V$ (see Section 4). We parametrize the space $\mathcal{Z}$ of Zygmund vector fields on $S^{1}$ that vanish at $1, i,-1 \in S^{1}$ in terms of the corresponding shear functions. Then we proceed to compute the Hilbert transform of Zygmund vector fields in terms of infinitesimal shear functions. We apply these formulas to compute the Weil-Petersson metric on Teichmüller spaces of finite punctured surfaces and the Fourier coefficients of Zygmund vector fields. Finally, we show that the formula for the Weil-Petersson metric in shear coordinates extend by continuity to the WeilPetersson metric of the boundary Teichmüller space in its shear coordinates (which is a result of Masur [18]). We give more details below.

A quasisymmetric map $h: S^{1} \rightarrow S^{1}$ induces a shear function $s: \mathcal{F} \rightarrow \mathbf{R}$ which assigns to each edge $e \in \mathcal{F}$ a real number

$$
s(e)=\log \operatorname{cr}(a, b, c, d)
$$

Date: October 29, 2018. 
where the endpoints of $e$ are $b, d \in S^{1}$, the two adjacent complementary triangles of $\mathcal{F}$ with common side $e$ have third vertices $a, c \in S^{1}$, and $\operatorname{cr}(a, b, c, d)=\frac{(b-c)(d-a)}{(b-a)(d-c)}$. A fan of geodesics of $\mathcal{F}$ with tip $p \in S^{1}$ is the set $\mathcal{F}_{p}$ of all geodesics of $\mathcal{F}$ with one endpoint $p$. Fix a correspondence of $\mathcal{F}_{p}$ with the integers $\mathbf{Z}$ such that $\mathcal{F}_{p}=\left\{e_{n}^{p}\right\}_{n \in \mathbf{Z}}$ and $e_{n}^{p}$ is adjacent to $e_{n+1}^{p}$ for all $n \in \mathbf{Z}$. We have the following characterization of quasisymmetric maps in terms of shear functions.

Theorem 1. [29] A shear function $s: \mathcal{F} \rightarrow \mathbf{R}$ induces a quasisymmetric map of the unit circle if and only if there exists a constant $M \geq 1$ such that for each fan $\mathcal{F}_{p}=\left\{e_{n}^{p}\right\}_{n \in \mathbf{Z}}$ and for each $m, k \in Z, k \geq 0$, we have

$$
\frac{1}{M} \leq e^{s\left(e_{m}\right)} \frac{1+e^{s\left(e_{m+1}\right)}+\cdots+e^{s\left(e_{m+1}\right)+\cdots+s\left(e_{m+k}\right)}}{1+e^{-s\left(e_{m-1}\right)}+\cdots+e^{-s\left(e_{m-1}\right)-\cdots-s\left(e_{m-k}\right)}} \leq M .
$$

A differentiable path $h_{t}: S^{1} \rightarrow S^{1},|t|<\epsilon$, of quasisymmetric maps with $h_{0}=i d$ induces a Zygmund vector field $V$ on $S^{1}$ by the formula

$$
V(z)=\left.\frac{d}{d t} h_{t}(z)\right|_{t=0}
$$

If $s_{t}: \mathcal{F} \rightarrow \mathbf{R}$ is the path of shear functions for $h_{t}$, then the derivative

$$
\left.\frac{d}{d t} s_{t}(e)\right|_{t=0}=\dot{s}(e)
$$

for $e \in \mathcal{F}$ induces a infinitesimal shear function

$$
\dot{s}: \mathcal{F} \rightarrow \mathbf{R}
$$

corresponding to the Zygmund vector field $V$. Our first result given in Theorem 4.1 is a characterization of infinitesimal shear functions which induce Zygmund bounded vector fields on $S^{1}$.

Theorem 2. Let $\dot{s}: \mathcal{F} \rightarrow \mathbf{R}$ be a shear function. Then $\dot{s}$ induces a Zygmund vector field $V$ on $S^{1}$ if and only if there exists a constant $C>0$ such that for all fans $\mathcal{F}_{p}=\left\{e_{n}^{p}\right\}_{n \in \mathbf{Z}}$ and for all $m, k \in \mathbf{Z}, k \geq 0$, we have

$$
\begin{aligned}
& \mid \dot{s}\left(e_{m}\right)+\frac{k}{k+1}\left[\dot{s}\left(e_{m+1}\right)+\dot{s}\left(e_{m-1}\right)\right]+\frac{k-1}{k+1}\left[\dot{s}\left(e_{m+2}\right)+\right. \\
& \left.\quad+\dot{s}\left(e_{m-2}\right)\right]+\cdots+\frac{1}{k+1}\left[\dot{s}\left(e_{m+k}\right)+\dot{s}\left(e_{m-k}\right)\right] \mid \leq C .
\end{aligned}
$$

A vector field on $S^{1}$ that vanishes at $1, i,-1$ is uniquely determined by its infinitesimal shear function. Therefore the above theorem gives an explicit (and rather simple) parametrization of the space $\mathcal{Z}$ (of all normalized Zygmund bounded vector fields) which is the tangent space at the basepoint of the universal Teichmüller space $T(\mathbf{H})$. This should be compared to the question of characterizing Zygmund vector fields (or Zygmund maps) in terms of its Fourier coefficients where no explicit condition is known (see [14]). The key idea in the proof of the above theorem is to "decompose" a vector field $V$ into an infinite sum of the vector fields $V_{p}$ over all fans of $\mathcal{F}_{p}$ of $\mathcal{F}$. The infinitesimal shear functions of $V_{p}$ are zero on all geodesics of $\mathcal{F}$ except on the geodesics of $\mathcal{F}_{p}$ where they are equal to (the one-half of) the 
infinitesimal shear function $\dot{s}$. We obtain (see Corollary 4.8)

$$
V(z)=\sum_{p \in \mathcal{F}^{0}} V_{p}(z)
$$

where $\mathcal{F}^{0}$ is the set of vertices of $\mathcal{F}$; the convergence is absolute and uniform; each partial sum of $V_{p}$ 's and the sum $V$ is Zygmund bounded with a Zygmund constant continuously depending on $C$. The decomposition of $V$ into vector fields $V_{p}$ corresponding to the fans of $\mathcal{F}$ is well-suited for the Zygmund vector fields, while writing the sum over all geodesics of $\mathcal{F}$ does not even produce a pointwise convergence on $S^{1}$. This is in an analogy with the Césaro summation of the Fourier series of a continuous function where the sum of the Fourier series does not necessarily converge at a single point of $S^{1}$ (see [14).

An interesting problem in Teichmüller theory is to express the complex structure of Teichmüller spaces in terms of the hyperbolic metric on the underlining surfaces. Kerckhoff proved that the Hilbert transform $H$ of the Zygmund vector fields on $S^{1}$ is the almost complex structure on the tangent space $\mathcal{Z}$ at the basepoint of the universal Teichmüller space $T(\mathbf{H})$ (see Nag-Verjovsky [20]). We compute the Hilbert transform in terms of infinitesimal shear functions (which are hyperbolic geometry invariants) which gives a solution to computing of the almost complex structure on the universal Teichmüller space $T(\mathbf{H})$ in terms of the hyperbolic geometry (see Theorem 5.2 and Theorem [5.4).

Theorem 3. Let $V$ be a Zygmund bounded vector field on $S^{1}$ and let $\dot{s}: \mathcal{F} \rightarrow \mathbf{R}$ be the corresponding shear function. Then the Hilbert transform of $V$ is given by the series

$$
H V(x)=\sum_{p \in \hat{\mathbf{Q}}} H V_{p}(x)
$$

where $H V_{p}$ is the Hilbert transform of $V_{p}$ and $V_{p}$ is defined as above using the shears in the fan $\mathcal{F}_{p}$. The series converges uniformly on $S^{1}$.

Moreover, the Hilbert transform $H\left(V_{p}\right)$ of the vector field $V_{p}$ corresponding to the fan $\mathcal{F}_{p}=\left\{e_{n}^{p}\right\}_{n \in \mathbf{Z}}$ is given by the formula

$$
H V_{p}(z)=\frac{1}{2} \sum_{n=-\infty}^{\infty} \dot{s}\left(e_{n}^{p}\right) \frac{\left(z-a_{n}^{p}\right)\left(z-b_{n}^{p}\right)}{a_{n}^{p}-b_{n}^{p}} \log \left|\frac{z-b_{n}^{p}}{z-a_{n}^{p}}\right|
$$

where $a_{n}^{p}$ is the initial point and $b_{n}^{p}$ is the terminal point of $e_{n}^{p}$.

Theorem 3 gives the Hilbert transform on Zygmund vector fields on $S^{1}$ in terms of infinitesimal shear functions from Theorem 2. Penner 24] gave a related formula for the Hilbert transform on $C^{5 / 2+\epsilon}$-smooth vector fields which are contained in the space of Zygmund vector fields. The formula in Theorem 3 is a double sum with the exterior sum over all all fans of $\mathcal{F}$ and the interior sum over all geodesic of a single fan. We remark that a single sum over all geodesics in $\mathcal{F}$ does not necessarily converge which forces the double sum. The formula of Theorem 3 can be explicitly given in terms of the hyperbolic geometry and we obtain an expression for the infinitesimal shear function $H(\dot{s})$ of the Zygmund bounded vector field $H(V)$ in terms of the hyperbolic invariants of $\mathcal{F}$ and the infinitesimal shear function $\dot{s}$ (see Corollary 6.1 and Corollary 6.2).

We also compute the Fourier coefficients of Zygmund bounded vector fields in terms of infinitesimal shear functions (see Theorem 7.1). 
Theorem 4. Let $V: S^{1} \rightarrow \mathbf{C}$ be a Zygmund vector field and let $\dot{s}: \mathcal{F} \rightarrow \mathbf{R}$ be the corresponding infinitesimal shear function. Then the $n$-th Fourier coefficient $\widehat{V}(n)$ of the vector field $V$ is given by

$$
\widehat{V}(n)=\sum_{p \in \mathcal{F}^{0}} \widehat{V}_{p}(n),
$$

where the convergence is absolute and

$$
\begin{array}{r}
\widehat{V}_{p}(n)=\sum_{e_{n}=\left(e^{i \phi_{0}^{n}}, e^{i \phi_{1}^{n}}\right) \in \mathcal{F}_{p}} \frac{\dot{s}\left(e_{n}\right)}{4 \pi\left(e^{i \phi_{0}}-e^{i \phi_{1}}\right)}\left[\frac{e^{i(2-n) \phi_{1}}-e^{i(2-n) \phi_{0}}}{i(2-n)}-\left(e^{i \phi_{0}}+e^{i \phi_{1}}\right) \times\right. \\
\left.\times \frac{e^{i(1-n) \phi_{1}}-e^{i(1-n) \phi_{0}}}{i(1-n)}+e^{i\left(\phi_{0}+\phi_{1}\right)} \frac{e^{-i n \phi_{1}}-e^{-i n \phi_{0}}}{-i n}\right]
\end{array}
$$

Finally, we consider the almost-complex structure on the Teichmüller space $T(S)$ of a finite area hyperbolic surface $S$ with at least one puncture. Let $\tau$ be an ideal geodesic triangulation of $S$ and let $\tilde{\tau}$ be the lift of $\tau$ to the hyperbolic plane $\mathbf{H}$. Then a infinitesimal shear function $\dot{\tilde{s}}: \tilde{\tau} \rightarrow \mathbf{R}$ which is invariant under the deck transformations for the universal covering of $S$ and which satisfies the cusp condition (see Section 8) represents a tangent vector $V$ to $T(S)$. We obtain a formula for the infinitesimal shear function $H(\dot{\tilde{s}})$ of the Hilbert transform of $V$ and for the Weil-Petersson metric on $T(S)$ (see Corollary 8.4 and Theorem 9.1).

Theorem 5. Let $\dot{\tilde{s}}: \tilde{\tau} \rightarrow \mathbf{R}$ be the infinitesimal shear function of a Zygmund bounded vector field $V$ invariant under a co-finite group $G$, where $\tilde{\tau}$ is an ideal geodesic triangulation of $\mathbf{H}$ invariant under $G$. Then the infinitesimal shear function

$$
H(\dot{\tilde{s}}): \tilde{\tau} \rightarrow \mathbf{R}
$$

of the vector field $H(V)$ obtained by taking the Hilbert transform of $V$ is given by

$$
H(\dot{\tilde{s}})((b, d))=\sum_{n \in \mathbf{N}} \dot{\tilde{s}}\left(e_{n}\right) \Delta_{b, d}\left(e_{n}\right)
$$

where $(b, d) \in \tilde{\tau}$ is the common boundary side of the two complementary triangles of $\tilde{\tau}$ with vertices $(a, b, d)$ and $(b, c, d), \tilde{\tau}=\left\{e_{n}\right\}_{n \in \mathbf{N}}$,

$$
\begin{aligned}
\Delta_{b, d}\left(e_{n}\right)= & \sinh ^{2}\left(\frac{\delta_{b, c}}{2}\right) \log \operatorname{coth}^{2}\left(\frac{\delta_{b, c}}{2}\right)+\cosh ^{2}\left(\frac{\delta_{a, d}}{2}\right) \log \operatorname{coth}^{2}\left(\frac{\delta_{a, d}}{2}\right)- \\
& \cosh ^{2}\left(\frac{\delta_{a, b}}{2}\right) \log \operatorname{coth}^{2}\left(\frac{\delta_{a, b}}{2}\right)-\cosh ^{2}\left(\frac{\delta_{c, d}}{2}\right) \log \operatorname{coth}^{2}\left(\frac{\delta_{c, d}}{2}\right)
\end{aligned}
$$

and $\delta_{b, c}$ is the distance between between the geodesic $e_{n}$ and the geodesic $(b, c)$ with analogous definition in other cases $\delta_{a, d}, \delta_{a, b}, \delta_{c, d}$. If $e_{n}$ has at least one endpoint in $(a, b, c, d)$ then $\Delta_{b, d}\left(e_{n}\right)$ is given appropriate interpretation as in Section 6.

Moreover, the Weil-Petersson metric pairing of the two tangent vectors $v_{1}, v_{2}$ to $T(S)$ represented by two infinitesimal shear functions $\dot{s}_{1}, \dot{s}_{2}: \tau \rightarrow \mathbf{R}$ is given by

$$
g_{W P}\left(v_{1}, v_{2}\right)=2 i\left(\dot{s}_{1}, H\left(\dot{s}_{2}\right)\right)
$$

where $H\left(\dot{s}_{2}\right)$ is the projection of $H\left(\dot{\tilde{s}}_{2}\right)$ onto $\tau$ and $i(\cdot, \cdot)$ is Thurston's algebraic intersection number.

We point out that the expression in the above theorem which represents the almost complex structure on the tangent space in terms of infinitesimal shear functions is given by a single sum unlike for arbitrary Zygmund bounded vector fields 
where the sum is double. This feature appears because the infinitesimal shear functions are invariant under the action of a cofinite Fuchsian group $G$. Finally, we use the above expression of the almost complex structure in terms of shear coordinates to conclude that the almost complex structure on $T(S)$ extend continuously to the boundary Teichmüller spaces of $T(S)$ obtained by pinching some closed curves on $S$ (see Section 10). Together with the result of J. Roger [27] which establishes that the Thurston's algebraic intersection number extend by continuity to the boundary of $T(S)$, we obtain the well-known result of Masur 18 that the Weil-Petersson metric extends by continuity to the boundary of $T(S)$. We also point out that G. Riera [26] obtained a formula for the Weil-Petersson metric on Teichmüller spaces of closed surfaces in terms of hyperbolic invariants.

\section{ZyGmund VeCtor FiELDS AND THE TANGENT SPACE to $T(\mathbf{H})$}

Let $\mathbf{H}=\{\operatorname{Im}(z)>0\}$ be the upper half-plane model of the hyperbolic plane with the hyperbolic metric given by $\rho(z)=\frac{|d z|}{\operatorname{Im}(z)}$. Then $\hat{\mathbf{R}}=\mathbf{R} \cup\{\infty\}$ is the ideal boundary of $\mathbf{H}$ and $\hat{\mathbf{R}}$ is homeomorphic to the unit circle $S^{1}$. A continuous map

$$
V: \mathbf{R} \rightarrow \mathbf{R}
$$

such that

$$
\lim _{x \rightarrow \infty} \frac{V(x)}{x^{2}}=\lim _{x \rightarrow-\infty} \frac{V(x)}{x^{2}}<\infty
$$

represents a (continuous) vector field on $\hat{\mathbf{R}}$. $\mathbf{R}$ is a chart of $\hat{\mathbf{R}}$ and the condition (1) guarantees that $V$ extends by continuity to a well-defined vector field at $\infty \in \hat{\mathbf{R}}$.

A vector field $V: \mathbf{R} \rightarrow \mathbf{R}$ on $\hat{\mathbf{R}}$ is said to be Zygmund if there exists $C>0$ such that both $V(x)$ and $x^{2} V(1 / x)$ satisfy

$$
|V(x+t)+V(x-t)-2 V(x)| \leq C t
$$

for $x \in[-2,2]$ and $t \in[0,1]$. If $V$ is Zygmund, then $V$ can be normalized by adding a quadratic polynomial in $x$ such that $V(0)=V(1)=0$ and $V(x)=O(x \log |x|)$ as $|x| \rightarrow \infty$ (see [11). We are mainly interested in Zygmund vector fields on $\hat{\mathbf{R}}$. A quadratic polynomial in $x$ describes an infinitesimal change in $P S L_{2}(\mathbf{R})$ which is a trivial change in our context of the universal Teichmüller space $T(\mathbf{H})$. Alternatively, a vector field $V: \mathbf{R} \rightarrow \mathbf{R}$ on $\hat{\mathbf{R}}$ is Zygmund if it satisfies (2) for all $x \in \mathbf{R}$ and $t>0$. The Zygmund norm of a Zygmund vector field $V: \mathbf{R} \rightarrow \mathbf{R}$ is the smallest constant $C$ for which (2) remains in force.

Let $a, b, c, d \in \hat{\mathbf{R}}$ be four distinct points. The cross-ratio $\operatorname{cr}(a, b, c, d)$ is defined by

$$
c r(a, b, c, d)=\frac{(c-b)(d-a)}{(b-a)(d-c)} .
$$

The cross-ratio norm $\|V\|_{c r}$ of a vector field $V: \mathbf{R} \rightarrow \mathbf{R}$ on $\hat{\mathbf{R}}$ is defined by

$$
\|V\|_{c r}=\sup _{c r(a, b, c, d)=1}\left|\frac{V(c)-V(b)}{c-b}+\frac{V(d)-V(a)}{d-a}-\frac{V(b)-V(a)}{b-a}-\frac{V(d)-V(c)}{d-c}\right| .
$$

A sequence $\left\{V_{n}\right\}_{n \in \mathbf{N}}$ of Zygmund vector fields converges to a Zygmund vector field $V$ in the topology of the Zygmund norm (the cross-ratio norm) if and only if the Zygmund norm (the cross-ratio norm) of $V-V_{n}$ goes to zero as $n \rightarrow \infty$. The 
convergence in the Zygmund norm is equivalent to the convergence in the cross-ratio norm [12, 11].

Gardiner and Sullivan [15, and Riemann [25] showed that a normalized vector field $V: \mathbf{R} \rightarrow \mathbf{R}$ is Zygmund if and only if there exists a differentiable path of quasisymmetric maps

$$
h_{t}: \hat{\mathbf{R}} \rightarrow \hat{\mathbf{R}}
$$

for $|t|<\epsilon$ and $\epsilon>0$ such that $h_{t}: 0,1, \infty \mapsto 0,1, \infty$ and

on $\mathbf{R}$.

$$
\left.\frac{d}{d t} h_{t}\right|_{t=0}=V
$$

We recall that the universal Teichmüller space $T(\mathbf{H})$ consists of all quasisymmetric maps $h: \hat{\mathbf{R}} \rightarrow \hat{\mathbf{R}}$ such that $h: 0,1, \infty \mapsto 0,1, \infty$. Thus the space $\mathcal{Z}$ of normalized Zygmund maps is identified with the tangent space $T_{i d} T(\mathbf{H})$ of the universal Teichmüller space $T(\mathbf{H})$ at the identity (basepoint) id $\in T(\mathbf{H})$.

\section{Complex structure on $T(\mathbf{H})$ and Hilbert transform on Zygmund VECTOR FIELDS}

Let $V: \mathbf{R} \rightarrow \mathbf{R}$ be a normalized Zygmund vector field $V$ on $\hat{\mathbf{R}}$, namely $V$ is a tangent vector to $T(\mathbf{H})$ at the basepoint $i d \in T(\mathbf{H})$. Let $\mu \in L^{\infty}(\mathbf{H})$ be a Beltrami differential representing the tangent vector $V \in T_{i d} T(\mathbf{H})$. Denote by $[\mu]$ the equivalence class of Beltrami differentials representing the same tangent vector as $\mu$. We have [3, 11] a standard formula

$$
V(x)=-\frac{2}{\pi} \operatorname{Re} \int_{\mathbf{H}} R(x, \zeta) \mu(\zeta) d \xi d \eta,
$$

where $R(x, \zeta)=\frac{x(x-1)}{\zeta(\zeta-1)(\zeta-x)}, \zeta=\xi+i \eta \in \mathbf{H}$ and $x \in \mathbf{R}$.

The complex structure operator $J: T_{i d} T(\mathbf{H}) \rightarrow T_{i d} T(\mathbf{H})$ is given by $J([\mu])=$ $[i \mu]$, where $[\mu]$ is the tangent vector represented by $\mu \in L^{\infty}(\mathbf{H})$ and $i \in \mathbf{C}$ is the imaginary unit. The above formula gives that the complex structure on $V$ is

$$
J(V)(x)=-\frac{2}{\pi} \operatorname{Re} \int_{\mathbf{H}} R(x, \zeta) i \mu(\zeta) d \xi d \eta
$$

or, equivalently

$$
J(V)(x)=\frac{2}{\pi} \operatorname{Im} \int_{\mathbf{H}} R(x, \zeta) \mu(\zeta) d \xi d \eta .
$$

Let $\tilde{V}(z)$ for $z \in \mathbf{H}$ be the Beurling-Ahlfors extension of $V$ into $\mathbf{H}$ (see [15, [11, pages 316,317$]$ ). Then we have that $\bar{\partial} \tilde{V}(z)=\mu(z)$ a.e. in $\mathbf{H}$ (see [11). By Stoke's theorem, we get

$$
\frac{2 i}{\pi} \int_{\mathbf{H}} R(x, \zeta) \mu(\zeta) d \xi d \eta=p \cdot v \cdot \frac{1}{\pi} \int_{\mathbf{R}} R(x, \xi) V(\xi) d \xi-i V(x) .
$$

By taking the real parts in the above equality, we obtain that the complex structure $J$ on $V$ is

$$
J(V)(x)=-\frac{1}{\pi} p \cdot v \cdot \int_{\mathbf{R}} R(x, \xi) V(\xi) d \xi
$$

for $x \in \mathbf{R}$ which equals the Hilbert transform of $V$

$$
H(V)(x)=-\frac{1}{\pi} p \cdot v \cdot \int_{\mathbf{R}} R(x, \xi) V(\xi) d \xi
$$


Kerckhoff was first to observe that the Hilbert transform gives the almostcomplex structure operator on $T_{i d} T(\mathbf{H})$ and the proof of this statement first appears in 20. We used the discussion in [10]. The Hilbert transform preserves the space of normalized Zygmund vector fields on $\hat{\mathbf{R}}$ (see [36, [10]).

Let $V_{(0, \infty)}: \mathbf{R} \rightarrow \mathbf{R}$ be the elementary shear vector field for the geodesic $(0, \infty)$ defined by

$$
V_{(0, \infty)}(x)= \begin{cases}x, & \text { for } x>0 \\ 0, & \text { for } x \leq 0\end{cases}
$$

Then the Hilbert transform of $V_{(0, \infty)}$ is given by

$$
H V_{(0, \infty)}(x)=\frac{1}{\pi} x \log |x|
$$

for all $x \in \mathbf{R}$.

Some more elementary integration gives

$$
\begin{aligned}
H\left(V_{(a, \infty)}\right)(x)= & \frac{1}{\pi}(x-a) \log |x-a|+\frac{(a-1) \log |a-1|}{\pi} x- \\
& -\frac{a \log a}{\pi}(x-1)
\end{aligned}
$$

for all $x \in \mathbf{R}$, where $a \in \mathbf{R}, a>0$, and the elementary shear vector field $V_{(a, \infty)}$ for the geodesic $(a, \infty)$ is defined by

$$
V_{(a, \infty)}(x)= \begin{cases}x-a, & \text { for } x>a \\ 0, & \text { for } x \leq a\end{cases}
$$

For $a \in \mathbf{R}, a<0$, the elementary shear vector field for the geodesic $(-\infty, a)$ is defined by

$$
V_{(-\infty, a)}(x)= \begin{cases}-(x-a), & \text { for } x<a \\ 0, & \text { for } x \geq a\end{cases}
$$

Then

$$
\begin{aligned}
H\left(V_{(-\infty, a)}\right)(x)= & -\frac{1}{\pi}(x-a) \log |x-a|-\frac{(a-1) \log |a-1|}{\pi} x+ \\
& +\frac{a \log |a|}{\pi}(x-1)
\end{aligned}
$$

Let $a, b \in \mathbf{R}$ and $a<b$. For the elementary shear vector field

$$
V_{(a, b)}(x)=\left\{\begin{array}{l}
\frac{(x-a)(x-b)}{a-b}, \text { for } a<x<b \\
0, \quad \text { otherwise }
\end{array}\right.
$$

we obtain the Hilbert transform

$$
\begin{aligned}
& H\left(V_{(a, b)}\right)(x)=\frac{1}{\pi} \frac{(x-a)(x-b)}{a-b} \log \left|\frac{x-b}{x-a}\right|- \\
& \quad-x \frac{(1-a)(1-b)}{\pi(a-b)} \log \left|\frac{b-1}{a-1}\right|+(x-1) \frac{a b}{\pi(a-b)} \log \left|\frac{b}{a}\right|
\end{aligned}
$$

for all $x \in \mathbf{R}$. 


\section{Shear Parametrization of Zygmund Maps}

Let $V: \mathbf{R} \rightarrow \mathbf{R}$ be a normalized Zygmund vector field. Namely $V(x)$ satisfies (2) and it vanishes at 0,1 and $\infty$, where vanishing at $\infty$ means that the rate of growth of $|V(x)|$ is $|x| \log |x|$ as $x \rightarrow \pm \infty$ (see [1] ). Let $h_{t}: \hat{\mathbf{R}} \rightarrow \hat{\mathbf{R}},|t|<\epsilon$, be a path of quasisymmetric maps such that $h_{0}=i d, h_{t}$ fixes 0,1 and $\infty$ for each $t$, $|t|<\epsilon$, and

$$
\left.\frac{d}{d t} h_{t}(x)\right|_{t=0}=V(x)
$$

for all $x \in \mathbf{R}$. In addition, we assume that the quasisymmetric constants of $h_{t}$ are bounded by $1+C t$ for some constant $C$ and for each $t,|t|<\epsilon$ (see [15], [1] ).

Given two ideal hyperbolic triangles $\Delta_{1}$ and $\Delta_{2}$ with common boundary side $e$ and disjoint interiors, the shear of $\Delta_{1}$ and $\Delta_{2}$ is the signed hyperbolic distance between the projections of third vertices of $\Delta_{2}$ and $\Delta_{1}$ onto $e$ with the orientation of $e$ as a part of the boundary of $\Delta_{1}$. A homeomorphism $h: \hat{\mathbf{R}} \rightarrow \hat{\mathbf{R}}$ maps the Farey tesselation $\mathcal{F}$ onto a tesselation $h(\mathcal{F})$. Given $e \in \mathcal{F}$, let $\Delta_{1}^{e}$ and $\Delta_{2}^{e}$ be the two complementary triangles of $h(\mathcal{F})$ with common boundary side $h(e)$. We define a shear function $s: \mathcal{F} \rightarrow \mathbf{R}$ of $h$ by setting $s(e)$ to be equal to the shear of the triangles $\Delta_{1}^{e}$ and $\Delta_{2}^{e}$.

Let $s_{t}: \mathcal{F} \rightarrow \mathbf{R}$ be the path of shear functions corresponding to the path $h_{t}$ of quasisymmetric maps. It follows that

$$
\dot{s}(e):=\left.\frac{d}{d t} s_{t}(e)\right|_{t=0}
$$

is well-defined for each $e \in \mathcal{F}$ by the differentiability of $h_{t}$. The function

$$
\dot{s}: \mathcal{F} \rightarrow \mathbf{R}
$$

associated to $V$ is called the infinitesimal shear function of $V$ and it uniquely determines $V$ when $V$ is normalized to vanish at 0,1 and $\infty$.

A fan of geodesics in $\mathcal{F}$ with tip $p \in \hat{\mathbf{Q}}$ consists of all geodesics of $\mathcal{F}$ with a common endpoint $p$. We orient each geodesic of the fan with tip $p$ such that $p$ is its initial point. Then for two geodesics $e$ and $e^{\prime}$ of the fan with tip $p$ we define $e<e^{\prime}$ if $e^{\prime}$ is to the left of $e$ and we define $e^{\prime}<e$, otherwise. This induces a one-to-one correspondence of the fan $\left\{e_{n}\right\}_{n \in \mathbf{Z}}$ with integers $\mathbf{Z}$ such that $e_{n}$ is adjacent to $e_{n+1}$ and $e_{n}<e_{n+1}$ for all $n \in \mathbf{Z}$. Under the above assumptions on $h_{t}$, there exists $C>0$ such that

$$
\frac{1}{1+C t} \leq e^{s_{t}(m)} \frac{1+e^{s_{t}(m+1)}+\cdots+e^{s_{t}(m+1)+\cdots+s_{t}(m+k)}}{1+e^{-s_{t}(m-1)}+\cdots+e^{-s_{t}(m-1)-\cdots-s_{t}(m-k)}} \leq 1+C t
$$

for all $m, k \in \mathbf{Z}$, where $s_{t}(m):=s_{t}\left(e_{m}\right)$ (see [29]). We have the expansion

$$
s_{t}(m)=\dot{s}(m) t+o_{m}(t)
$$

where $o_{m}(t) / t \rightarrow 0$ as $t \rightarrow 0$ and constant in $o_{m}(t)$ depends on $m$. From (9) and the above expansion, we obtain

$$
\begin{aligned}
\mid \dot{s}(m) & +\frac{k-1}{k}[\dot{s}(m+1)+\dot{s}(m-1)]+\frac{k-2}{k}[\dot{s}(m+2)+ \\
& +\dot{s}(m-2)]+\cdots+\frac{1}{k}[\dot{s}(m+k)+\dot{s}(m-k)] \mid \leq C
\end{aligned}
$$

for all $m, k \in \mathbf{Z}$, for all fans $\left\{e_{n}\right\}_{n \in \mathbf{Z}}$ of $\mathcal{F}$ and for the fixed constant $C>0$ from the above. Thus (10) is a necessary condition for $\dot{s}: \mathcal{F} \rightarrow \mathbf{R}$ to be the infinitesimal shear 
function of a Zygmund map $V$. We show that (10) is also a sufficient condition. Thus the space $\mathcal{Z}$ of all Zygmund vector fields normalized to vanish at 0,1 and $\infty$ is parameterized by the set of functions $\dot{s}: \mathcal{F} \rightarrow \mathbf{R}$ which satisfy (10) in all fans for some constant $C>0$ (which is the same for all fans).

Theorem 4.1. Let $\dot{s}: \mathcal{F} \rightarrow \mathbf{R}$ be an arbitrary function. Then $\dot{s}$ is the infinitesimal shear function of a normalized Zygmund vector field $V: \mathbf{R} \rightarrow \mathbf{R}$ if and only if $\dot{s}$ satisfies (10) for all fans in $\mathcal{F}$ with a fixed constant $C$.

Proof. We proved above that (10) is a necessary condition for $V$ to be a Zygmund vector field. It remains to prove that (10) is also a sufficient condition for $\dot{s}$ to be induced by a Zygmund vector field $V: \mathbf{R} \rightarrow \mathbf{R}$. We first show that $\dot{s}$ is induced by a continuous vector field $V: \mathbf{R} \rightarrow \mathbf{R}$.

Let $\Delta_{0}$ be a hyperbolic triangle in the upper half-plane $\mathbf{H}$ with vertices 0,1 and $\infty$. We orient each edge in $\mathcal{F}$ to the left as seen from $\Delta_{0}$. Consider a fan of geodesics $\mathcal{F}_{p}=\left\{e_{n}^{p}\right\}_{n \in \mathbf{Z}}$ with tip $p \in \hat{\mathbf{Q}}$. The geodesics of the fan $\mathcal{F}_{p}$ are divided into two groups with respect to the orientation given by $\Delta_{0}$ : the geodesics whose initial point is $p$ and the geodesics whose terminal point is $p$. We enumerate the fan with tip $p$ by $\left\{e_{n}^{p}\right\}_{n \in \mathbf{Z}}$ such that $e_{n}^{p}$ is adjacent to $e_{n+1}^{p}$, and that $e_{0}^{p}$ has initial point $p$ and $e_{1}^{p}$ has terminal point $p$. This enumeration is unique. In the case $p=\infty$, we have that $e_{n}^{\infty}$ is a geodesic with endpoints $n$ and $\infty$.

Assume that function

$$
\dot{s}: \mathbf{Z} \rightarrow \mathbf{R}
$$

satisfies (10). We define

$$
V_{\dot{s}}^{\infty}(x)= \begin{cases}0 & ; x \in[0,1] \\ \dot{s}(1)(x-1)+\cdots+\dot{s}(n)(x-n) & ; x \in(n, n+1] \\ -\dot{s}(0) x-\cdots-\dot{s}(-n)(x+n) & ; x \in[-n-1,-n)\end{cases}
$$

for $n \in \mathbf{N}$.

Proposition 4.2. Under the above notation, the vector field $V_{\dot{s}}^{\infty}: \mathbf{R} \rightarrow \mathbf{R}$ is Zygmund with the Zygmund norm $2 C+18\|\dot{s}\|_{\infty} \leq 20 C$, where $C$ is given in (10).

Proof. It is clear that $V_{\dot{s}}^{\infty}$ is continuous. We consider $x \in \mathbf{R}$ and $t \geq 2$. Let $m \in \mathbf{Z}$ and $k \in \mathbf{N}$ be such that $m \leq x \leq m+1$ and $m+k \leq x+t \leq m+k+1$. Then we have $k-1 \leq t \leq k+1$ and $m-k-1 \leq x-t \leq m-k+2$. A simple estimation shows that

$$
\begin{array}{r}
\left|V_{\dot{s}}^{\infty}(x+t)+V_{\dot{s}}^{\infty}(x-t)-2 V_{\dot{s}}^{\infty}(x)\right| \leq \mid V_{\dot{s}}^{\infty}(m+k)+V_{\dot{s}}^{\infty}(m-k)- \\
-2 V_{\dot{s}}^{\infty}(m) \mid+6\|\dot{s}\|_{\infty} k+3\|\dot{s}\| \leq C_{1} t
\end{array}
$$

where $C_{1}=2 C+18\|\dot{s}\|_{\infty}$ because $k \geq 1$ implies $2 t \geq k$ and condition (10) is equivalent to $\left|V_{\dot{s}}^{\infty}(m+k)+V_{\dot{s}}^{\infty}(m-k)-2 V_{\dot{s}}^{\infty}(m)\right| \leq C k$.

For $0<t<2$, we have that $V_{\dot{s}}^{\infty}$ is piecewise linear on each interval $[x-t, x+t]$ with at most three points where it is not differentiable and with the bound $\|\dot{s}\|_{\infty}$ on the difference between the two adjacent slopes. Thus $\mid V_{\dot{s}}^{\infty}(x+t)+V_{\dot{s}}^{\infty}(x-$ $t)-2 V_{\dot{s}}^{\infty}(x) \mid \leq C_{1} t$ in this case as well. Thus $V_{\dot{s}}^{\infty}$ is Zygmund bounded with the Zygmund norm $C_{1}$.

Continuation of the proof of Theorem 4.1. We introduce an order on $\hat{\mathbf{Q}}$ using the Farey tesselation. Namely, 0 and $\infty$ in $\hat{\mathbf{Q}}$ are said to be of order 1 . The numbers 
1 and -1 are said to be of order 2. If the maximum of the orders of $a / b$ and $c / d$ is $n$ and they are adjacent on $\hat{\mathbf{R}}$ (when we consider only numbers in $\hat{\mathbf{Q}}$ of order at most $n)$, then the mediant $(a+c) /(b+d)$ is of the order $n+1$. In this fashion, we obtain the whole $\hat{Q}$. There are two order 1 and two order 2 numbers in $\hat{Q}$. For $n \geq 2$, there are $2^{n-1}$ numbers of order $n$.

The reference triangle $\Delta_{0}$ has vertices 0,1 and $\infty$. Fix $p \in \hat{\mathbf{Q}}$ and let $\mathcal{F}_{p}=$ $\left\{e_{n}^{p}\right\}_{n \in \mathbf{Z}}$ be the fan with tip $p$. Recall that $e_{n}^{p}$ 's are oriented to the left as seen from $\Delta_{0}$, and that $e_{0}^{p}$ has the initial point $p$ and $e_{1}^{p}$ has the terminal point $p$. Let $a$ be the terminal endpoint of $e_{0}^{p}$ and let $B \in P S L_{2}(\mathbf{Z})$ be the unique element such that $B(0)=a$ and $B(\infty)=p$. Let $V_{\dot{s}_{p}}^{\infty}$ be given by formula (11) where

$$
\dot{s}_{p}: \mathbf{Z} \rightarrow \mathbf{R}
$$

is defined by

$$
\dot{s}_{p}(n)=\frac{1}{2} \dot{s}\left(e_{n}^{p}\right)
$$

for $n \in \mathbf{Z}$. (Note that we use $\frac{1}{2} \dot{s}$ because each geodesic of $\mathcal{F}$ appears in two different fans.) Then $V_{\dot{s}_{p}}^{\infty}$ is a Zygmund vector field with the Zygmund constant $10 C$ by Proposition 4.2 .

Define

$$
V_{p}:=B^{*} V_{\dot{s}_{p}}^{\infty}
$$

Then $V_{p}$ is a Zygmund vector field corresponding to the shear function which agrees with $\frac{1}{2} \dot{s}$ at $\mathcal{F}_{p}=\left\{e_{n}^{p}\right\}_{n \in \mathbf{Z}}$ and which is zero on $\mathcal{F} \backslash \mathcal{F}_{p}$. If $p \neq 0,1$ then $V_{p}$ is normalized to be zero on $(-\infty, B(0)) \cup(B(1),+\infty)$ if $B(0)<B(1)$, otherwise $V_{p}$ is zero on $(-\infty, B(1)) \cup(B(0),+\infty)$ if $B(1)<B(0)$. In both cases $V_{p}$ vanishes at $\infty$. It is also true that $V_{p}$ vanishes at 0 and 1 because geodesics in $\mathcal{F}_{p}$ do not separate 0 and 1 from $\Delta_{0}$. Thus $V_{p}$ is a normalized Zygmund vector field.

We define a formal series

$$
V(x):=\sum_{p \in \hat{\mathbf{Q}}} V_{p}(x)
$$

for $x \in \mathbf{R}$. It is clear that $V$ is well-defined on $\hat{\mathbf{Q}}$ because only finitely many terms $V_{p}(x)$ in the above series are non-zero. (If we knew that $V$ is a tangent vector to some path of homeomorphisms, then the corresponding infinitesimal shear function would be equal to $\dot{s}: \mathcal{F} \rightarrow \mathbf{R}$. However, we still need to show that $V$ is defined on all of $\mathbf{R}$ and that it is Zygmund.)

Proposition 4.3. The series

$$
V(x):=\sum_{p \in \hat{\mathbf{Q}}} V_{p}(x)
$$

converges absolutely and uniformly on compact subsets of $\mathbf{R}$.

Proof. Let $x \in \mathbf{R}$. By Proposition 4.2 and the above, the Zygmund norm of each $V_{\dot{s}_{p}}^{\infty}$ is bounded by $C_{1}=10 C$. This implies that the cross-ratio norm of each $V_{\dot{s}_{p}}^{\infty}$ is bounded by $C_{2}=C_{2}\left(C_{1}\right)$ (see [15, [11]). Thus the Zygmund norm of $V_{b}$ is bounded by $C_{2}$ because $V_{p}$ is normalized to vanish at 0,1 and $\infty$.

Let $r_{x}$ be the geodesic ray from the center of $\Delta_{0}$ to $x \in \mathbf{R}$. Consider the set of fans whose geodesics intersect $r_{x}$. If $r_{x}$ intersects $\mathcal{F}_{p}$ and the Farey order of $p$ is at least 2 , then $r_{x}$ does not intersect any other fan with the same order. On the other 
hand, $r_{x}$ intersects both $\mathcal{F}_{0}$ and $\mathcal{F}_{\infty}$ if and only if $r_{x}$ intersects the geodesic with endpoints 0 and $\infty$. We order the set of all fans intersecting $r_{x}$ by the Farey order of their tips. The only ambiguity is if both $\mathcal{F}_{0}$ and $\mathcal{F}_{\infty}$ intersect $r_{x}$ in which case we set $\mathcal{F}_{0}<\mathcal{F}_{\infty}$. This gives us a linear order on the set of fans intersecting $r_{x}$ and we can put them in a sequence $\left\{\mathcal{F}_{p_{n}}\right\}_{n \in \mathbf{N}}$ such that $\mathcal{F}_{p_{n}}<\mathcal{F}_{p_{n+1}}$. Consider the ordered subset $\left\{e_{i_{1}}^{p_{n}}, \ldots, e_{i_{k(n)}}^{p_{n}}\right\}$ of geodesics of $\mathcal{F}_{p_{n}}$ that intersect $r_{x}$. Then either $i_{1}=0$ and $i_{1}>i_{2}>\cdots>i_{k}$, or $i_{1}=1$ and $i_{1}<i_{2}<\cdots<i_{k}$.

Consider the fans $\mathcal{F}_{p_{n}}$ and $\mathcal{F}_{p_{n+2}}$. The last geodesic $e_{i_{k(n)}}^{p_{n}}$ of the fan $\mathcal{F}_{p_{n}}$ which intersects $r_{x}$ does not share an endpoint with the first geodesic $e_{i_{1}}^{p_{n+2}}$ of the fan $\mathcal{F}_{p_{n+2}}$ which intersects $r_{x}$. Thus the distance between $e_{i_{k(n)}}^{p_{n}}$ and $e_{i_{1}}^{p_{n+2}}$ is $\delta=\log (1+\sqrt{2})^{2}$.

Assume that $n \geq 3$. Then no geodesic of the fan $\mathcal{F}_{p_{n}}$ has $\infty$ as an endpoint. Moreover, the Farey order of $p_{n}$ is at least 3 . Let $e_{i_{1}}^{p_{n}}=\left(a_{n}, b_{n}\right)$ be the first geodesic of the fan $\mathcal{F}_{p_{n}}$ that intersects $r_{x}$. Note that $V_{p_{n}}\left(a_{n}\right)=V_{p_{n}}\left(b_{n}\right)=0$ by the normalization of $V_{p_{n}}$. The distance of the center of the triangle $\Delta_{0}$ to $e_{i_{1}}^{p_{n}}$ is at least $\frac{n-2}{2} \delta$ by the above considerations. An elementary hyperbolic geometry implies that $\left|a_{n}-b_{n}\right|$ is of the order $e^{-\frac{(n-2) \delta}{2}}$ for $n \in \mathbf{N}$, where the constant depends on a compact subset of $\mathbf{R}$ in which $x$ lies. Since $V_{p_{n}}$ has $\epsilon \log \frac{1}{\epsilon}$ modulus of continuity with the constant depending on its Zygmund norm and since the support of $V_{p_{n}}$ is the interval in $\mathbf{R}$ with endpoints $a_{n}$ and $b_{n}$, for $x$ in a compact subset of $\mathbf{R}$, we obtain

$$
\sum_{p \in \hat{\mathbf{Q}}}\left|V_{p}(x)\right|=\sum_{n \in \mathbf{N}}\left|V_{p_{n}}(x)\right| \leq C_{0}+C_{3} \sum_{n \in \mathbf{N}} n e^{-\frac{n \delta}{2}}<\infty
$$

where $C_{0}=\left|V_{p_{1}}(x)\right|+\left|V_{p_{2}}(x)\right|+\left|V_{p_{3}}(x)\right|$, and $C_{3}=C_{3}\left(C_{2}\right)$ is a constant which depends on the bound $C_{2}$ on the Zygmund norm of $V_{p_{n}}$ 's.

Remark 4.4. We showed that the formal series (12) converges to a continuous vector field on $\mathbf{R}$ which is normalized to vanish at 0 and 1 because each $V_{p}$ vanishes at 0 and 1 . To see that $V(x)$ extends to a (continuous) normalized vector field on $\hat{\mathbf{R}}$ we need to show that $V(x)=o\left(x^{2}\right)$ as $x \rightarrow \pm \infty$. In the proof of Proposition 4.3 we showed that $V(x)$ (for $x$ in a compact subset of $\mathbf{R}$ ) is bounded in terms of the Zygmund bound on $V_{p}$ 's. The push-forward of the vector fields $V_{p}$ under the translation $T: x \mapsto x+a, a \in \mathbf{R}$, satisfies $\left\|V_{p}\right\|_{\infty}=\left\|T^{*}\left(V_{p}\right)\right\|_{\infty}$ because $T^{*}\left(V_{p}\right)(x)=V_{p}(x-a)$. Let $\sum^{\prime} V_{p_{n}}(x)$ be the sum (12) without terms $V_{p_{1}}, V_{p_{2}}$ and $V_{p_{3}}$. Note that each $V_{p_{n}}$ in the sum $\sum^{\prime} V_{p_{n}}(x)$ does not have a positive shear on a geodesic of $\mathcal{F}$ with one endpoint $\infty$. If $x \in[k, k+1]$ for $k \in \mathbf{Z}$, define $T_{k}(x)=x-k$. Then $\left(T_{k}\right)^{*}\left(\sum^{\prime} V_{p_{n}}(x)\right)$ is equal to $V_{k}^{\prime}(x-k)$, where the infinitesimal shear function of the vector field $V_{k}^{\prime}$ is the push-forward by $T_{k}$ of infinitesimal shear function $\dot{s}$. Since $V_{k}^{\prime}$ is bounded on compact subsets of $\mathbf{R}$ by Proposition 4.3. since $x-k \in[0,1]$ and since $\sum^{\prime} V_{p_{n}}(x)=V_{k}^{\prime}(x-k)$, it follows that $\sum^{\prime} V_{p_{n}}(x)$ is bounded on $\mathbf{R}$. Since $V_{p_{1}}, V_{p_{2}}$ and $V_{p_{3}}$ are normalized to vanish at $\infty$ and $\sum^{\prime} V_{p_{n}}(x)$ is bounded on $\mathbf{R}$, it follows that $V(x)=o\left(x^{2}\right)$ as $x \rightarrow \pm \infty$. Thus (12) defines a normalized vector field on $\hat{\mathbf{R}}$.

Remark 4.5. The sum (12) defines a continuous vector field $V: \mathbf{R} \rightarrow \mathbf{R}$ on $\hat{\mathbf{R}}$ which vanishes at 0,1 and $\infty$. This is achieved by normalizing each vector field $V_{b}$ in the same fashion. To normalize $V(x)$ to vanish at the endpoints $a_{1}, a_{2}, a_{3}$ of another complementary triangle $\Delta$ of $\mathcal{F}$, we start the construction of each $V_{p}$ with 
respect to the triangle $\Delta$. Equivalently, we need to add a quadratic polynomial to the formula (12) in order to obtain a vector field with the same infinitesimal shear function $\dot{s}$ which vanishes at $a_{1}, a_{2}, a_{3}$. The new vector field is of the order $O\left(x^{2}\right)$ as $x \rightarrow \pm \infty$.

Remark 4.6. Let $B \in P S L_{2}(\mathbf{R})$ and let $\mathcal{F}^{*}=B(\mathcal{F})$. Then $\dot{s} \circ B^{-1}: \mathcal{F}^{*} \rightarrow \mathbf{R}$ is a shear function on $\mathcal{F}^{*}$. Fix a complementary triangle $\Delta^{*}$ in $\mathcal{F}^{*}$. Then there exists $B_{1} \in P S L_{2}(\mathbf{R})$ such that $B_{1}\left(\Delta_{0}\right)=\Delta^{*}$ and $B_{1}(\mathcal{F})=\mathcal{F}^{*}$. We define

$$
W(x):=\sum_{p \in B_{1}(\hat{\mathbf{Q}})} W_{p}(x)
$$

where $W_{p}=\left(B_{1}\right)^{*}\left(V_{B_{1}^{-1}(p)}\right)$. Then the series for $W(x)$ converges absolutely and uniformly on compact subsets of $\mathbf{R} \backslash\left\{B_{1}(\infty)\right\}$ because each term is the push-forward of the series (12) by the same Möbius map $B_{1} \in P S L_{2}(\mathbf{R})$. Vector field $W: \mathbf{R} \rightarrow \mathbf{R}$ is normalized to vanish at the vertices of $\Delta^{*}$ and its infinitesimal shear function is $\dot{s} \circ B_{1}^{-1}: \mathcal{F}^{*} \rightarrow \mathbf{R}$.

We continue the proof of Theorem 4.1. Proposition 4.3 implies that $V(x)$ is a continuous vector field on $\mathbf{R}$ because each $V_{p}(x)$ is a continuous vector field and the convergence is uniform. We showed in Remark 4.4 that $V(x)$ extends to a normalized vector field on $\hat{\mathbf{R}}$. We need to show that $V(x)$ is a Zygmund vector field. We show a slightly stronger statement which will be used later on.

Proposition 4.7. Let $C>0$ be fixed. If

$$
\dot{s}: \mathcal{F} \rightarrow \mathbf{R}
$$

satisfies (10) with constant $C$, then there exists $M=M(C)>0$ such that the vector field

$$
V: \mathbf{R} \rightarrow \mathbf{R}
$$

induced by $\dot{s}$ is $M(C)$-Zygmund bounded.

Proof. Assume on the contrary that there exists a sequence $\dot{t}_{n}: \mathcal{F} \rightarrow \mathbf{R}$ of infinitesimal shear functions satisfying (10) with the fixed constant $C>0$ such that the cross-ratio norms of the corresponding vector fields $W_{n}: \mathbf{R} \rightarrow \mathbf{R}$ converge to infinity as $n \rightarrow \infty$. Then there exists a sequence $\left\{Q_{n}=\left(a_{n}, b_{n}, c_{n}, d_{n}\right)\right\}$ of quadruples on $\hat{\mathbf{R}}$ with $\operatorname{cr}\left(Q_{n}\right)=\frac{\left(c_{n}-a_{n}\right)\left(d_{n}-b_{n}\right)}{\left(d_{n}-a_{n}\right)\left(c_{n}-b_{n}\right)}=2$ such that $\left|W_{n}\left[Q_{n}\right]\right| \rightarrow \infty$ as $n \rightarrow \infty$. (If for a single shear function $\dot{t}: \mathcal{F} \rightarrow \mathbf{R}$ the induced continuous vector field $W$ is not Zygmund, then there exists a sequence $Q_{n}$ as above such that $\left|W\left[Q_{n}\right]\right| \rightarrow \infty$ as $n \rightarrow \infty$. We replace $W_{n}$ with $W$ and the argument below is the same.) We seek a contradiction.

Let $Q^{*}:=\left(-e^{\delta},-1,1, e^{\delta}\right)$ with $\delta=\log (1+\sqrt{2})^{2}$ and note that $\operatorname{cr}\left(Q^{*}\right)=2$. Then there exists a unique Möbius map $B_{n}$ such that $B_{n}\left(Q_{n}\right)=Q^{*}$. Define

$$
V_{n}:=\left(B_{n}\right)^{*} W_{n} .
$$

Then

$$
\left|V_{n}\left[Q^{*}\right]\right| \rightarrow \infty
$$

as $n \rightarrow \infty$.

Recall that $V_{n}(x)=\frac{1}{\left(B_{n}^{-1}\right)^{\prime}(x)} W_{n}\left(B_{n}^{-1}(x)\right)$. Let $\mathcal{F}_{n}=B_{n}(\mathcal{F})$ and $\dot{s}_{n}:=\dot{t}_{n} \circ B_{n}^{-1}$. Then $V_{n}$ is a vector field corresponding to $\dot{s}_{n}$ whose support is an ideal triangulation 
$\mathcal{F}_{n}$ and which is normalized to be zero at the vertices of the complementary triangle $\Delta_{n}:=B_{n}\left(\Delta_{0}\right)$. There are two possibilities to consider:

(a) $\mathcal{F}_{n}$ converges to an ideal triangulation $\mathcal{F}^{*}$ of $\mathbf{H}$, or

(b) $\mathcal{F}_{n}$ does not converge to an ideal triangulation of $\mathbf{H}$.

In both cases we are allowed to further normalize the sequence $V_{n}$ to vanish at any three points on $\hat{\mathbf{R}}$ by adding a quadratic polynomial.

Assume we are in the case (a). Then the sequence $\dot{s}_{n}$ of infinitesimal shear functions has a convergent subsequence in the weak*-topology (if we consider $\dot{s}_{n}$ as functions on the space of geodesics on $\mathbf{H}$ ). Without loss of generality, we assume that $\dot{s}_{n}$ converges to $\dot{s}^{*}$ as $n \rightarrow \infty$. The support of $\dot{s}^{*}$ is $\mathcal{F}^{*}$. Moreover, $\dot{s}^{*}$ satisfies property (10) in each fan of $\mathcal{F}^{*}$ because the values of $\dot{s}^{*}$ in each fan of $\mathcal{F}^{*}$ are limits of the values of $\dot{s}_{n}$ on the fans of $\mathcal{F}_{n}$ (and all $\dot{s}_{n}$ satisfy property (10) with the same constant). Note that all shears of $\mathcal{F}^{*}$ are 0 because it is the limit of $\mathcal{F}_{n}$ whose each shear is 0 . Thus $\mathcal{F}^{*}$ is the image of the Farey tesselation under a Möbius map $B^{*} \in P S L_{2}(\mathbf{R})$. By Remark 4.6, the infinitesimal shear function $\dot{s}^{*}$ determines a continuous vector field $V^{*}: \mathbf{R} \rightarrow \mathbf{R}$. If the sequence $V_{n}$ pointwise converges to $V^{*}$ then we get a contradiction with $\left|V_{n}\left[Q^{*}\right]\right| \rightarrow \infty$ because $\left|V_{n}\left[Q^{*}\right]\right| \rightarrow\left|V^{*}\left[Q^{*}\right]\right|<\infty$ as $n \rightarrow \infty$.

To obtain a contradiction in the case (a), it is enough to prove the convergence of $V_{n}$ to $V^{*}$. By Remark 4.6, we have

$$
V^{*}(x)=\sum_{p \in \mathcal{F}_{0}^{*}} V_{p}^{*}(x)
$$

for $x \in \mathbf{R}$, where $\mathcal{F}_{0}^{*}$ is the set of vertices of $\mathcal{F}^{*}$. The convergence is absolute and uniform on compact subsets of $\mathbf{R}$. Recall that each $V_{p}^{*}$ is normalized to be zero on the vertices of $\Delta_{n}:=B_{n}\left(\Delta_{0}\right)$. Let $\Delta^{*}$ be the limit of $\Delta_{n}$. If the sequence $\Delta_{n}$ does not converge, then we normalize $V_{n}$ to be zero on the vertices $\Delta_{n}^{\prime}$ such that the sequence $\Delta_{n}^{\prime}$ converges. We denote the normalization of $V_{n}$ by $V_{n}$ again and $\Delta_{n}^{\prime}$ by $\Delta_{n}$ for simplicity of notation. Then we normalize $V^{*}(x)$ to be zero at the vertices of the complementary triangle $\Delta^{*}$ of $\mathcal{F}^{*}$ by normalizing each $V_{p}^{*}$ to be zero at these points.

Given a compact subset $K$ of $\mathbf{R}$ and an $\epsilon>0$, there exists finitely many $p_{1}, p_{2}, \ldots, p_{k} \in \mathcal{F}_{0}^{*}$ such that

$$
\left|V^{*}(x)-\sum_{i=1}^{k} V_{p_{i}}^{*}(x)\right|<\epsilon
$$

for all $x \in K$. To see this, consider the sequence of all fans $\mathcal{F}_{p_{n}}^{*}$ which intersect all rays $r_{x}$ from the center of $\Delta^{*}$ to $x \in K$. Given $d>0$, there exists $k$ such that the support of $V_{p_{i}}^{*}$ for $i>k$ has length smaller than $d$. (This was established for the Farey tesselation $\mathcal{F}$ and the proof is similar for $\mathcal{F}^{*}$.) The lefthand side of (13) is less than

$$
\sum_{i=k+1}^{\infty}\left|V_{p_{i}}^{*}(x)\right| \leq C \sum_{i=k+1}^{\infty} i e^{-\frac{i \delta}{2}}
$$

where $C$ is given in the last paragraph in the proof of Proposition 4.3 . The estimate (13) follows for $k$ large enough.

For each fan $\mathcal{F}_{p_{i}}^{*}, i=1,2, \ldots, k$, there exists a sequence of fans $\left\{\left(\mathcal{F}_{n}\right)_{p_{i}^{n}}\right\}_{n \in \mathbf{N}}$ which converges to $\mathcal{F}_{p_{i}}^{*}$ as $n \rightarrow \infty$ such that the functions $\left.\dot{s}_{n}\right|_{\left(\mathcal{F}_{n}\right)_{p_{i}^{n}}}$ converge in the 
weak* topology to $\left.\dot{s}_{\infty}\right|_{\mathcal{F}_{p_{i}}^{*}}$. We have

$$
\left|V_{n}(x)-\sum_{i=1}^{k}\left(V_{n}\right)_{p_{i}^{n}}(x)\right|<\sum_{i=k+1}^{\infty}\left|\left(V_{n}\right)_{p_{i}^{n}}(x)\right|<C_{n} \sum_{i=k+1}^{\infty} i e^{-\frac{i \delta}{2}}
$$

where $C_{n}$ is the constant corresponding to $V_{n}$ similar to the above.

Note that $\left\{C_{n}\right\}_{n \in \mathbf{N}}$ is a bounded sequence. Then

$$
\left|V_{n}(x)-\sum_{i=1}^{k}\left(V_{n}\right)_{p_{i}^{n}}(x)\right|<\epsilon
$$

for all $x \in K$ and $n$ large enough. Thus $k$ can be chosen simultaneously for $V^{*}$ and all $V_{n}, n \in \mathbf{N}$ depending only on $\epsilon>0$.

By (13) and (15), it is enough to prove that $\left(V_{n}\right)_{p_{i}^{n}}(x) \rightarrow V_{p_{i}}^{*}(x)$ uniformly for $x$ in the compact subset $K$ of $\mathbf{R}$ as $n \rightarrow \infty$. Note that $\left(V_{n}\right)_{p_{i}^{n}}$ and $V_{p_{i}}^{*}$ are Zygmund vector fields with uniformly bounded cross-ratio norms and the supports of $\left(V_{n}\right)_{p_{i}^{n}}$ on $\mathbf{R}$ converge to the support of $V_{p_{i}}^{*}$. Thus the uniform convergence of $\left(V_{n}\right)_{p_{i}^{n}}$ to $V_{p_{i}}^{*}$ follows from the pointwise convergence on a dense subset of the compact subset $K$ of $\mathbf{R}$ because $\left\{\left(V_{n}\right)_{p_{i}^{n}}\right\}$ is a normal family. On the other hand, the weak* convergence of $\left.\dot{s}_{n}\right|_{\left(\mathcal{F}_{n}\right)_{p_{i}^{n}}}$ to $\left.\dot{s}_{\infty}\right|_{\mathcal{F}_{p_{i}}^{*}}$ implies that $\left(V_{n}\right)_{p_{i}^{n}}$ converges pointwise to $V_{p_{i}}^{*}$, when $\left(V_{n}\right)_{p_{i}^{n}}$ are normalized to vanish at the vertices of the triangle $\Delta_{n}$ in $\mathcal{F}_{n}$ with $\Delta_{n} \rightarrow \Delta^{*}$ as $n \rightarrow \infty$. Thus $V_{n}$ converges uniformly to $V^{*}$ on $K$ and case (a) is finished.

It remains to consider the case (b). The sequence $\mathcal{F}_{n}$ has a convergent subsequence in the Hausdorff topology on closed subset of $\mathbf{H}$. Without loss of generality we assume that the whole sequence $\mathcal{F}_{n}$ converges to a subset $\mathcal{F}^{*}$ of the space of geodesics of $\mathbf{H}$. The limit $\mathcal{F}^{*}$ of $\mathcal{F}_{n}$ is a geodesic lamination of $\mathbf{H}$ because each $\mathcal{F}_{n}$ is a geodesic lamination. Moreover, $\mathcal{F}^{*}$ is not empty because there is a bounded neighborhood of the imaginary unit $i \in \mathbf{H}$ which has to intersect an edge of $\mathcal{F}_{n}$ (since complementary triangles of $\mathcal{F}_{n}$ cover $\mathbf{H}$ and there is a positive upper bound on the distance of points inside an ideal triangle to its boundary). If $\mathcal{F}^{*}$ has a non-empty complement, then the connected components of the complement consist of ideal triangles because the connected components of the complement of $\mathcal{F}_{n}$ are ideal triangles. However, if a connected component of the complement of $\mathcal{F}^{*}$ is an ideal triangle then $\mathcal{F}^{*}$ contains all the images of the triangle under the group of isometries of $\mathbf{H}$ generated by inversions in the sides of the triangle. This implies that $\mathcal{F}^{*}$ is an ideal triangulation which contradicts the case (b). Thus $\mathcal{F}^{*}$ is necessarily a geodesic foliation of $\mathbf{H}$.

Let $l$ be a leaf of the foliation $\mathcal{F}^{*}$ which contains $i \in \mathbf{H}$ and let $\Delta_{n}$ be a complementary triangle of $\mathcal{F}_{n}$ which contains $i \in \mathbf{H}$. Two boundary sides of $\Delta_{n}$ converge to $l$ as $n \rightarrow \infty$. Let $p_{n} \in \hat{\mathbf{R}}$ be the common endpoint of the above two boundary sides. Recall that

with

$$
V_{n}:=\left(B_{n}\right)^{*}\left(W_{n}\right)
$$

$$
\left|V_{n}\left[Q^{*}\right]\right| \rightarrow \infty
$$

as $n \rightarrow \infty$. By Proposition 4.3 and Remark 4.6 we have

$$
V_{n}(x)=\left(V_{n}\right)_{p_{n}}(x)+\sum_{p^{\prime} \in\left(B_{n}(\hat{Q}) \backslash\left\{p_{n}\right\}\right)}\left(V_{n}\right)_{p^{\prime}}(x) .
$$


Note that $V_{n}$ vanishes at vertices of $B_{n}\left(\Delta_{0}\right)$, where $\Delta_{0}$ is the ideal triangle with vertices 0,1 and $\infty$. We normalize $V_{n}$ by adding a quadratic polynomial such that it vanishes at the vertices of $\Delta_{n}$, where two boundary sides of $\Delta_{n}$ converge to $l \in \mathcal{F}^{*}$. We denote by $V_{n}^{\prime}$ the normalized vector field. Then we have

$$
V_{n}^{\prime}(x)=\left(V_{n}\right)_{p_{n}}^{\prime}(x)+\sum_{p^{\prime} \in\left(B_{n}(\hat{Q}) \backslash\left\{p_{n}\right\}\right)}\left(V_{n}\right)_{p^{\prime}}^{\prime}(x)
$$

where each $\left(V_{n}\right)_{p^{\prime}}^{\prime}$ is defined to vanish at the vertices of the triangle $\Delta_{n}$. Note that $\dot{s}_{n}=\dot{t}_{n} \circ B_{n}^{-1}$ is the infinitesimal shear function of $V_{n}^{\prime}$ and $V_{n}$. Since the cross-ratio norm is invariant under the addition of a quadratic polynomial, we have

$$
\left|V_{n}^{\prime}\left[Q^{*}\right]\right| \rightarrow \infty
$$

as $n \rightarrow \infty$.

Since the reference triangle $\Delta_{n}$ converges to a single geodesic $l$, it follows that all geodesics of $\mathcal{F}_{n}$ except the geodesics in the fan with tip $p_{n}$ have the supremum of their Euclidean sizes going to zero as $n \rightarrow \infty$. Then, similar to the proof of Proposition 4.3 we have

$$
\sum_{p^{\prime} \in\left(B_{n}(\hat{Q}) \backslash\left\{p_{n}\right\}\right)}\left|\left(V_{n}\right)_{p^{\prime}}^{\prime}(x)\right| \leq C \sum_{i=1}^{\infty}(a(n)+i) e^{\frac{-(a(n)+i) \delta}{2}}
$$

with $a(n) \rightarrow \infty$ as $n \rightarrow \infty$. Thus $\sum_{p^{\prime} \in\left(B_{n}(\hat{Q}) \backslash\left\{p_{n}\right\}\right)}\left|\left(V_{n}\right)_{p^{\prime}}^{\prime}(x)\right|$ is arbitrary small when $n$ is large. By Proposition 4.2. $\left(V_{n}\right)_{p_{n}}^{\prime}(x)$ are Zygmund bounded with the cross-ratio norms independent of $n$. We further normalize $\left(V_{n}\right)_{p_{n}}^{\prime}(x)$ by adding a quadratic polynomial $q_{n}(x)$ such that $\left(V_{n}\right)_{p_{n}}^{\prime \prime}(x):=\left(V_{n}\right)_{p_{n}}^{\prime}(x)+q_{n}(x)$ vanishes at 0,1 and $\infty$. It follows that $\left\{\left(V_{n}\right)_{p_{n}}^{\prime \prime}\right\}_{n \in \mathbf{N}}$ is a normal family. Thus there exists a subsequence of $\left\{\left(V_{n}\right)_{p_{n}}^{\prime \prime}\right\}_{n \in \mathbf{N}}$ which converges uniformly. Without loss of generality, we assume that $\left(V_{n}\right)_{p_{n}}^{\prime \prime}$ converges to a continuous function $V_{*}^{\prime \prime}$ uniformly on compact subsets of $\mathbf{R}$.

Define $V_{n}^{\prime \prime}:=V_{n}^{\prime}+q_{n}$. Then $V_{n}^{\prime \prime} \rightarrow V_{*}^{\prime \prime}$ as $n \rightarrow \infty$ uniformly on compact subsets of $\mathbf{R}$ by the above discussion. This implies that $\left|V_{n}\left[Q^{*}\right]\right|=\left|V_{n}^{\prime}\left[Q^{*}\right]\right|=\left|V_{n}^{\prime \prime}\left[Q^{*}\right]\right| \rightarrow$ $\left|V_{*}^{\prime \prime}\left[Q^{*}\right]\right|<\infty$ as $n \rightarrow \infty$. This contradicts $\left|V_{n}\left[Q^{*}\right]\right| \rightarrow \infty$ as $n \rightarrow \infty$. Thus case (b) cannot occur as well. It follows that the sequence $\left\{V_{n}(x)\right\}_{n \in \mathbf{N}}$ has uniformly bounded Zygmund norms.

End of the proof of Theorem 4.1. Proposition 4.3 states that $\dot{s}$ is induced by a continuous vector field $V$. Proposition 4.7] states that $V$ is Zygmund bounded with the Zygmund constant depending on the constant $C$ in (10) which finishes the proof.

The proof of the above theorem also establishes the uniform convergence property of the series defining the Zygmund vector field in terms of the infinitesimal shear function $\dot{s}: \mathcal{F} \rightarrow \mathbf{R}$. The series giving the vector field corresponding to the infinitesimal shear function $\dot{s}: \mathcal{F} \rightarrow \mathbf{R}$ is defined by adding over all fans vector fields corresponding to the fans which is a particular order of the summation of the series of elementary shear vector fields for $\dot{s}$. This is analogous to the convergence of the Césaro sum of the Fourier series of continuous functions on $S^{1}$ (see [14]).

Note that there exist continuous functions on the unit disk $S^{1}$ whose Fourier series does not converge at a single point of $S^{1}$. Similarly, the series $V(x)=$ 
$\sum_{e=(a, b) \in \mathcal{F}} \dot{s}(e) \chi_{[a, b]}(x) \frac{(x-a)(x-b)}{a-b}$, where $\chi_{[a, b]}$ is the characteristic function of the interval $[a, b]$, does not necessarily converge on $\mathbf{R} \backslash \hat{\mathbf{Q}}$ because the infinitesimal shear function $\dot{s}$ does not induce a bounded measured lamination in the sense of Thurston (see [30, 28). Thus we need a summation method similar to the situation for the Fourier series which is given in the following corollary.

Corollary 4.8. Let $\dot{s}: \mathcal{F} \rightarrow \mathbf{R}$ be a shear function which satisfies (10) in each fan of $\mathcal{F}$. Then the series defining the corresponding vector field

$$
V(x)=\sum_{p \in \hat{\mathbf{Q}}} V_{p}(x)
$$

converges absolutely and uniformly on compact subsets of $\mathbf{R}$, where $V_{p}(x)$ is the vector field corresponding to $\left.\dot{s}\right|_{\mathcal{F}_{p}}$. The term $V_{p}(x)$ is a piecewise quadratic polynomial except at the tip $p$. Moreover, we have

$$
\left|V(x)-\sum_{i=1}^{k} V_{p_{i}}(x)\right|<C \sum_{i=n}^{\infty} i e^{-\frac{(i-2) \delta}{2}}
$$

where $\delta=\log (1+\sqrt{2})^{2}, C$ is a function of the constant in (10) and $\left\{p_{1}, \ldots, p_{k}\right\}$ are all Farey number of order at most $n$. In addition, the Zygmund norms of $V(x)$ and $\sum_{i=1}^{k} V_{p_{i}}(x)$ for $k \in \mathbf{N}$ are bounded by constant $M(C)$, where $C$ is the constant from (10).

\section{The Hilbert transform in Shears}

Let $V: \mathbf{R} \rightarrow \mathbf{R}$ be a Zygmund vector field on $\hat{\mathbf{R}}$ and let $\dot{s}: \mathcal{F} \rightarrow \mathbf{R}$ be the corresponding infinitesimal shear function. We use the formula

$$
V(x)=\sum_{p \in \hat{Q}} V_{p}(x)
$$

to find the Hilbert transform of $V$ in terms of the corresponding infinitesimal shear function $\dot{s}$, where the order of the summation is given by increasing Farey orders of tips $p \in \hat{\mathbf{Q}}$. Recall that the fan $\mathcal{F}_{p}$ with tip $p$ is enumerated by $\left\{e_{n}\right\}_{n \in \mathbf{Z}}$ such that the initial point of $e_{0}$ is $p$ and the terminal point of $e_{1}$ is $p$, where $e_{n}$ is oriented to the left as seen from the reference triangle $\Delta_{0}$. We define the tip-p infinitesimal shear function $\dot{s}_{p}: \mathcal{F}_{p}=\left\{e_{n}\right\}_{n \in \mathbf{N}} \rightarrow \mathbf{R}$ by setting $\dot{s}_{p}\left(e_{n}\right):=\frac{1}{2} \dot{s}\left(e_{n}\right)$. Thus $\dot{s}_{p}$ satisfies (10) with the constant $\frac{1}{2} C$, where $C$ is the constant of $\dot{s}$. Therefore

$$
V_{p}(x)=\sum_{n \in \mathbf{Z}} \dot{s}_{p}\left(e_{n}\right) V_{\left(a_{n}, b_{n}\right)}(x)
$$

is Zygmund bounded with the cross-ratio norm bounded independently of the fan $\mathcal{F}_{p}$, where $e_{n}$ has initial point $a_{n}$ and terminal point $b_{n}$, and $V_{\left(a_{n}, b_{n}\right)}$ is the elementary shear vector field defined in section 3 .

Since $V_{p}$ is Zygmund bounded, it follows that the Hilbert transform $H V_{p}$ is welldefined and also Zygmund bounded (see [36, 10, [11). Moreover, the cross-ratio norm of $H V_{p}$ is bounded in terms of the cross-ratio norm of $V_{p}$.

We first give a lemma which facilitates various convergence arguments in the rest of the paper. 
Lemma 5.1. Let $V_{n}: \mathbf{R} \rightarrow \mathbf{R}$, for $n \in \mathbf{N}$, and $V: \mathbf{R} \rightarrow \mathbf{R}$ be Zygmund bounded vector fields on $\hat{\mathbf{R}}$ normalized to vanish at 0,1 and $\infty$. Suppose that

$$
V_{n}(x) \rightarrow V(x)
$$

as $n \rightarrow \infty$ uniformly on compact subsets of $\mathbf{R}$ and that the sequence $\left\{V_{n}\right\}_{n \in \mathbf{N}}$ has uniformly bounded cross-ratio norms. Then

$$
H\left(V_{n}\right)(x) \rightarrow H(V)(x)
$$

as $n \rightarrow \infty$ uniformly on compact subsets of $\mathbf{R}$.

Proof. Recall that the Hilbert transform of $V(x)$ is given by

$$
H V(x)=-\frac{1}{\pi} \lim _{\epsilon \rightarrow 0}\left[\int_{-\infty}^{x-\epsilon} \frac{x(x-1)}{\zeta(\zeta-1)(\zeta-x)} V(\zeta) d \zeta+\int_{x+\epsilon}^{\infty} \frac{x(x-1)}{\zeta(\zeta-1)(\zeta-x)} V(\zeta) d \zeta\right] .
$$

Since $V, V_{n}$ are normalized Zygmund vector fields with uniformly bounded crossratio norms, it follows that there exists $M>0$ such that $|V(x)|,\left|V_{n}\right| \leq M|x| \log |x|$ as $|x| \rightarrow \infty$ (see [11]). Consequently, $\int_{R}^{\infty} \frac{x(x-1)}{\zeta(\zeta-1)(\zeta-x)} V(\zeta) d \zeta, \int_{R}^{\infty} \frac{x(x-1)}{\zeta(\zeta-1)(\zeta-x)} V_{n}(\zeta) d \zeta$, $\int_{-\infty}^{-R} \frac{x(x-1)}{\zeta(\zeta-1)(\zeta-x)} V(\zeta) d \zeta$ and $\int_{-\infty}^{-R} \frac{x(x-1)}{\zeta(\zeta-1)(\zeta-x)} V_{n}(\zeta) d \zeta$ are of the order $o\left(R^{-\alpha}\right)$ for some $0<\alpha<1$ (see [1]). Since $V_{n}$ converges to $V$ uniformly on compact subsets of $\mathbf{R}$, it follows that (for a fixed $\epsilon>0$ )

$$
\int_{-\infty}^{x-\epsilon} \frac{V(\zeta)-V_{n}(\zeta)}{\zeta(\zeta-1)(\zeta-x)} d \zeta+\int_{x+\epsilon}^{\infty} \frac{V(\zeta)-V_{n}(\zeta)}{\zeta(\zeta-1)(\zeta-x)} d \zeta \rightarrow 0
$$

as $n \rightarrow \infty$.

To finish the proof, it is enough to show that for any $\delta>0$ there exists $\epsilon=$ $\epsilon(\delta)>0$ and $n_{0}=n_{0}(\delta) \in \mathbf{N}$ such that

$$
\left|p . v . \int_{x-\epsilon}^{x+\epsilon} \frac{x(x-1)}{\zeta(\zeta-1)(\zeta-x)}\left[V(\zeta)-V_{n}(\zeta)\right] d \zeta\right|<\delta
$$

for all $n \geq n_{0}$

Let $\tilde{V}$ and $\tilde{V}_{n}$ be the Beurling-Ahlfors extensions to $\mathbf{H}$ of $V$ and $V_{n}$ respectively (see [15]). The $\bar{\partial}$-derivatives $\mu$ and $\mu_{n}$ of $\tilde{V}$ and $\tilde{V}_{n}$ are Beltrami differentials on $\mathbf{H}$ corresponding to the vector fields $V$ and $V_{n}$ (see [15], 11]). The vector fields $V$ and $V_{n}$ have uniformly (in $n$ ) bounded cross-ratio norms which implies the existence of a constant $S>0$ such that $\|\mu\|_{\infty},\left\|\mu_{n}\right\|_{\infty}<S$ for all $n \in \mathbf{N}$. Since $V_{n}$ converges to $V$ uniformly on compact subsets of $\mathbf{R}$, it follows that $\tilde{V}_{n}$ converges to $\tilde{V}$ uniformly on compact subsets of $\mathbf{H} \cup \mathbf{R}$ when considered as a subset of $\mathbf{C}$. Stoke's theorem gives

$$
\begin{gathered}
p . v . \int_{x-\epsilon}^{x+\epsilon} \frac{x(x-1)}{\zeta(\zeta-1)(\zeta-x)} V(\zeta) d \zeta=\iint_{D_{\epsilon}(x)} \frac{x(x-1) \mu(\xi)}{\xi(\xi-1)(\xi-x)} d \zeta d \eta- \\
-\int_{C_{\epsilon}(x)} \frac{x(x-1)}{\xi(\xi-1)(\xi-x)} \tilde{V}(\xi) d \xi
\end{gathered}
$$

where $\mu=\bar{\partial} \tilde{V}, D_{\epsilon}(x)$ is the upper half-disk with center $x$ and radius $\epsilon$, and $C_{\epsilon}(x)$ is the circular part of the boundary of $D_{\epsilon}(x)$. A similar equation holds for $V_{n}$.

By (17), to prove (16) it is enough to prove that

$$
\iint_{D_{\epsilon}(x)}\left|\frac{x(x-1) \mu_{n}(\xi)}{\xi(\xi-1)(\xi-x)}\right| d \zeta d \eta \rightarrow 0
$$


as $\epsilon \rightarrow 0$ uniformly in $n \in \mathbf{N}$, and that

$$
\int_{C_{\epsilon}(x)} \frac{x(x-1)}{\xi(\xi-1)(\xi-x)}\left[\tilde{V}(\xi)-\tilde{V}_{n}(\xi)\right] d \xi \rightarrow 0
$$

as $n \rightarrow \infty$ for all $\epsilon<1$. Since $\left\|\mu_{n}\right\|_{\infty} \leq S$, and the expression $\frac{x(x-1)}{\xi(\xi-1)(\xi-x)}$ has a simple pole at $x$, and the area of $D_{\epsilon}(x)$ goes to zero as $\epsilon \rightarrow 0$, we obtain (18).

To obtain (19), we change the variable $\xi=x+\epsilon e^{i \varphi}$ to obtain

$$
\begin{aligned}
\int_{C_{\epsilon}(x)} & \frac{\left[\tilde{V}(\xi)-\tilde{V}_{n}(\xi)\right]}{\xi(\xi-1)(\xi-x)} d \xi= \\
=\int_{0}^{\pi} & \frac{\left[\tilde{V}\left(x+\epsilon e^{i \varphi}\right)-\tilde{V}_{n}\left(x+\epsilon e^{i \varphi}\right)\right]}{\left(x+\epsilon e^{i \varphi}\right)\left(x-1+\epsilon e^{i \varphi}\right) \epsilon e^{i \varphi}} \epsilon i e^{i \varphi} d \varphi= \\
& =\int_{0}^{\pi} \frac{\left[\tilde{V}\left(x+\epsilon e^{i \varphi}\right)-\tilde{V}_{n}\left(x+\epsilon e^{i \varphi}\right)\right]}{\left(x+\epsilon e^{i \varphi}\right)\left(x-1+\epsilon e^{i \varphi}\right)} i d \varphi
\end{aligned}
$$

The last integral converges to zero because $\tilde{V}_{n} \rightarrow \tilde{V}$ uniformly on compact subsets of $\mathbf{H} \cup \mathbf{R}$.

In the following theorem we decompose the Hilbert transform $H V$ into an infinite sum of the Hilbert transforms $H V_{p}$ for $p \in \hat{\mathbf{Q}}$ analogous to Corollary 4.8, The main tool in the proof is Lemma 5.1 .

Theorem 5.2. Let $V: \mathbf{R} \rightarrow \mathbf{R}$ be a Zygmund vector field on $\hat{\mathbf{R}}$ and let $\dot{s}: \mathcal{F} \rightarrow \mathbf{R}$ be the corresponding infinitesimal shear function. Then the Hilbert transform of $V$ is given by the series

$$
H V(x)=\sum_{p \in \hat{\mathbf{Q}}} H V_{p}(x)
$$

where $H V_{p}$ is the Hilbert transform of $V_{p}$ and $V_{p}$ is defined as above using the shears in the fan $\mathcal{F}_{p}$. The series converges uniformly on compact subsets of $\mathbf{R}$.

Remark 5.3. We point out that the uniform convergence of the series is strong property because the Hilbert transform is given by the principal value of an improper integral. Moreover, the infinitesimal shear function has variable sign which further complicates the convergence. On the other hand, it seems that the series does not converge absolutely. One should also note that the "naive" series $H V(x)=\sum_{e=(a, b) \in \mathcal{F}} \dot{s}(e) H V_{(a, b)}(x)$ does not converge, where $H V_{(a, b)}(x)$ is given by (8), (7), (6), or (5).

Proof. Let $V_{n}(x)=\sum_{i=1}^{k} V_{p_{i}}(x)$ such that $\left\{p_{1}, p_{2}, \ldots, p_{k}\right\}$ is the set of Farey numbers of order at most $n$. Then $V_{n}$ converges to $V$ uniformly on compact subsets of $\mathbf{R}$ by Corollary 4.8. Proposition 4.7 states that each $V_{n}$ is a Zygmund vector field with uniformly bounded Zygmund norms. The theorem follows from Lemma 5.1 .

We compute the Hilbert transform $H V_{p}$ of $V_{p}$ in terms of shears.

Theorem 5.4. Let $\dot{s}_{p}: \mathcal{F}_{p} \rightarrow \mathbf{R}$ be a shear function that satisfies (10) in the fan $\mathcal{F}_{p}=\left\{e_{n}\right\}_{n \in \mathbf{Z}}$ and let $V_{p}$ be the induced vector field. Then

$$
H V_{p}(x)=\sum_{n=-\infty}^{\infty} \dot{s}_{p}\left(e_{n}\right) H\left(V_{e_{n}}\right)(x)
$$


where $V_{e_{n}}$ is the elementary shear vector field for the geodesic $e_{n}$ oriented to the left as seen from the reference triangle $\Delta_{0}$.

Proof. We divide the proof into two cases $p=\infty$ and $p \neq \infty$.

Assume first that $p=\infty$. We have

$$
\begin{aligned}
& V_{\infty}(\zeta)=\dot{s}_{\infty}(1)(\zeta-1)+\dot{s}_{\infty}(2)(\zeta-2)+\cdots+ \\
& +\dot{s}_{\infty}(n)(\zeta-n)=\left(\sum_{i=1}^{n} \dot{s}_{\infty}(i)\right) \zeta-\sum_{i=1}^{n} i \dot{s}_{\infty}(i)
\end{aligned}
$$

for $1 \leq n \leq \zeta \leq n+1$, we have

$$
V_{\infty}(x)=0
$$

for $0 \leq \zeta \leq 1$, and we have

$$
\begin{gathered}
V_{\infty}(\zeta)=\dot{s}_{\infty}(0) \zeta+\dot{s}_{\infty}(-1)(\zeta+1)+\cdots+ \\
+\dot{s}_{\infty}(-n)(\zeta+n)=\left(\sum_{i=0}^{n} \dot{s}_{\infty}(-i)\right) \zeta+\sum_{i=0}^{n} i \dot{s}_{\infty}(-i)
\end{gathered}
$$

for $-n-1 \leq \zeta \leq-n \leq 0$.

Since $V_{\infty}$ is a Zygmund map, it follows that $H\left(V_{\infty}\right)$ exists and it is also a Zygmund map. Thus

$$
-\frac{1}{\pi} \int_{-n}^{n} \frac{x(x-1)}{\zeta(\zeta-1)(\zeta-x)} V_{\infty}(\zeta) d \zeta \rightarrow H\left(V_{\infty}\right)(x)
$$

as $n \rightarrow \infty$ for each $x \in \mathbf{R}$. Define

$$
V_{\infty}^{n}(x)= \begin{cases}V_{\infty}(x), & \text { for }-n \leq x \leq n \\ \left(\sum_{i=1}^{n} \dot{s}_{\infty}(i)\right) x-\sum_{i=1}^{n} i \dot{s}_{\infty}(i), & \text { for } x>n \\ \left(\sum_{i=0}^{n} \dot{s}_{\infty}(-i)\right) x+\sum_{i=0}^{n} i \dot{s}_{\infty}(-i), & \text { for } x<-n\end{cases}
$$

It follows that

$$
-\frac{1}{\pi} \int_{-n}^{n} \frac{x(x-1)}{\zeta(\zeta-1)(\zeta-x)} V_{\infty}(\zeta) d \zeta=-\frac{1}{\pi} \int_{-n}^{n} \frac{x(x-1)}{\zeta(\zeta-1)(\zeta-x)} V_{\infty}^{n}(\zeta) d \zeta .
$$

Since the Hilbert transform is given by an integral kernel, it follows that

$$
H\left(V_{\infty}^{n}\right)(x)=\sum_{i=-n}^{n} \dot{s}_{\infty}(i) H\left(V_{e_{i}}\right)(x) .
$$

To finish the proof of the theorem in this case, it is enough to show that

$$
\int_{n}^{\infty} \frac{x(x-1)}{\zeta(\zeta-1)(\zeta-x)} V_{\infty}^{n}(\zeta) d \zeta \rightarrow 0
$$

and

as $n \rightarrow \infty$.

$$
\int_{-\infty}^{-n} \frac{x(x-1)}{\zeta(\zeta-1)(\zeta-x)} V_{\infty}^{n}(\zeta) d \zeta \rightarrow 0
$$

Using elementary computations, we obtain

$$
\begin{aligned}
& \int_{n}^{\infty} \frac{x(x-1)}{\zeta(\zeta-1)(\zeta-x)} V_{\infty}^{n}(\zeta) d \zeta= \\
& x(x-1) \int_{n}^{\infty} \frac{\left(\sum_{i=-n}^{n} \dot{s}_{\infty}(i)\right) \zeta-\sum_{i=-n}^{n} i \dot{s}_{\infty}(i)}{\zeta(\zeta-1)(\zeta-x)} d \zeta= \\
& \left(\sum_{i=-n}^{n} \dot{s}_{\infty}(i)\right) O\left(\frac{1}{n}\right)+\left(\sum_{i=-n}^{n} i \dot{s}_{\infty}(i)\right) O\left(\frac{1}{n^{2}}\right)
\end{aligned}
$$


If the tip- $\infty$ infinitesimal shear function which satisfies (10) in addition satisfies

$$
\sum_{i=-n}^{n} \dot{s}_{\infty}(i)=o(n)
$$

then

$$
\sum_{i=-n}^{n} i \dot{s}_{\infty}(i)=o\left(n^{2}\right)
$$

and the quantity (22) converges to zero as $n \rightarrow \infty$. Thus, to finish the proof that $\int_{n}^{\infty} \frac{x(x-1)}{\zeta(\zeta-1)(\zeta-x)} V_{\infty}^{n}(\zeta) d \zeta \rightarrow 0$ as $n \rightarrow \infty$ it remains to show (23) which is done in Lemma 5.5 below. The proof of $\int_{-\infty}^{-n} \frac{x(x-1)}{\zeta(\zeta-1)(\zeta-x)} V_{\infty}^{n}(\zeta) d \zeta \rightarrow 0$ as $n \rightarrow \infty$ is similar and left to the reader. This finishes the proof for $p=\infty$.

Assume that $p=0$ and let $\mathcal{F}_{0}=\left\{e_{n}\right\}_{n \in \mathbf{Z}}$ be the fan with tip 0 . Then $e_{1}=(0, \infty)$ and $e_{n}=\left(0, \frac{1}{-n+1}\right)$ for $n \in \mathbf{Z} \backslash\{1\}$. Fix $n \in \mathbf{N}$. We have

$$
V_{0}^{n}(\zeta)=\dot{s}\left(e_{0}\right) \frac{\zeta(\zeta-1)}{1}+\dot{s}\left(e_{-1}\right) \frac{\zeta\left(\zeta-\frac{1}{2}\right)}{\frac{1}{2}}+\cdots+\dot{s}\left(e_{-n+1}\right) \frac{\zeta\left(\zeta-\frac{1}{n}\right)}{\frac{1}{n}}
$$

for $0 \leq \zeta \leq \frac{1}{n}$. A short computation gives

$$
V_{0}^{n}(\zeta)=\left[\sum_{i=1}^{n} i \dot{s}(-i+1)\right] \zeta^{2}-\left[\sum_{i=1}^{n} \dot{s}(-i+1)\right] \zeta
$$

for $0 \leq \zeta \leq \frac{1}{n}$

By the above and by Lemma (5.5), we get that

$$
\left|V_{0}^{n}(\zeta)\right| \leq o\left(n^{2}\right) \zeta^{2}+o(n) \zeta
$$

for $0 \leq \zeta \leq \frac{1}{n}$. Then

$$
\left|\int_{0}^{1 / n} \frac{V_{0}^{n}(\zeta)}{\zeta(\zeta-1)(\zeta-x)} d \zeta\right| \leq o\left(n^{2}\right) \frac{1}{n^{2}}+o(n) \frac{1}{n} \rightarrow 0
$$

as $n \rightarrow \infty$. Similarly

$$
\left|\int_{-1 / n}^{0} \frac{V_{0}^{n}(\zeta)}{\zeta(\zeta-1)(\zeta-x)} d \zeta\right| \rightarrow 0
$$

as $n \rightarrow \infty$. This implies $H\left(V_{0}^{n}\right) \rightarrow H\left(V_{0}\right)$ as $n \rightarrow \infty$.

Assume that $p \neq \infty, 0$. We have that

$$
\left|V_{p}^{n}(\zeta)\right| \leq o\left(n^{2}\right)(\zeta-p)^{2}+o(n)|\zeta-p|
$$

because the vector field $V_{p}^{n}$ is the push-forward by a Möbius map $B: 0 \mapsto p$ of the vector field $V_{0}^{n}$. The argument that

$$
\int_{p}^{p_{n+1}} \frac{V_{p}^{n}(\zeta)}{\zeta(\zeta-1)(\zeta-x)} \rightarrow 0
$$

as $n \rightarrow \infty$ proceeds similarly as the case $p=0$ when $x=p$, and it is even easier when $x \neq p$ because the integrand does not have a singularity for $n$ large. Thus $H\left(V_{p}^{n}\right) \rightarrow H\left(V_{p}\right)$ as $n \rightarrow \infty$.

We obtained that $H\left(V_{p}^{n}\right) \rightarrow H\left(V_{p}\right)$. Since $H\left(V_{p}^{n}\right)(x)=\sum_{i=-n}^{n} \dot{s}_{p}\left(e_{i}\right) H\left(V_{e_{i}}\right)(x)$ the theorem follows. 
Lemma 5.5. Let $\dot{s}: \mathcal{F} \rightarrow \mathbf{R}$ be a shear function which satisfies (10) in a fan $\left\{e_{n}\right\}_{n \in \mathbb{Z}}$. Then

$$
\sum_{i=k}^{k+n} \dot{s}\left(e_{i}\right)=o(n)
$$

for each $k \in \mathbb{Z}$.

Proof. Let $a_{0}=\dot{s}\left(e_{k}\right)$ and $a_{i}=a_{i-1}+\dot{s}\left(e_{k+i}\right)+\dot{s}\left(e_{k-i}\right)$ for $i \geq 1$. Then the condition (10) becomes

$$
\left|\frac{1}{n}\left(a_{0}+a_{1}+\cdots+a_{n-1}\right)\right| \leq C .
$$

From (10) we also obtain

$$
\left|a_{i}-a_{i-1}\right| \leq 2 C
$$

for all $i \geq 1$. The statement of the lemma translates to $a_{n}=o(n)$.

Assume on the contrary that there exists a sequence $i_{n} \rightarrow \infty$ as $n \rightarrow \infty$ and a constant $k>0$ such that

$$
\left|a_{i_{n}}\right| \geq k i_{n}
$$

for all $n$. Without loss of generality, we assume that

$$
a_{i_{n}} \geq k i_{n}
$$

and seek a contradiction. By (25), we get that

$$
\begin{aligned}
& a_{i_{n}+1} \geq a_{i_{n}}-2 C \geq k i_{n}-2 C \\
& a_{i_{n}+2} \geq a_{i_{n}}-4 C \geq k i_{n}-4 C \\
& \cdots \\
& a_{i_{n}+j} \geq a_{i_{n}}-2 j C \geq k i_{n}-2 j C
\end{aligned}
$$

for some $j \in \mathbb{Z}$. Adding the inequalities in (26), we obtain

$$
a_{0}+a_{1}+\cdots+a_{i_{n}}+a_{i_{n}+1}+\cdots+a_{i_{n}+j} \geq k i_{n} j-j(j+1) C-C i_{n} .
$$

If $j=\left[\sqrt{i_{n}}\right]$ then

$$
\frac{1}{i_{n}+j}\left(a_{0}+\cdots a_{i_{n}+j}\right) \geq \frac{k i_{n} j}{i_{n}+j}-\frac{j(j+1)}{i_{n}+j} C-\frac{i_{n} C}{i_{n}+j} \rightarrow \infty
$$

as $n \rightarrow \infty$. This is in a contradiction with (24).

\section{RECOVERING THE SHEARS}

We gave a formula for the Hilbert transform of a Zygmund vector field in terms of the corresponding infinitesimal shear function (see theorems 5.2 and 5.4). We describe below how to obtain the infinitesimal shear function $\dot{s}$ corresponding to a vector field $V: \mathbf{R} \rightarrow \mathbf{R}$.

Let $a, b, c, d \in \hat{\mathbf{R}}$ (given in the counter-clockwise order) be four vertices of an ideal hyperbolic quadrilateral which is decomposed into the union of the triangle with vertices $a, b, d$ and the triangle with vertices $b, c, d$. Then the value of the shear function $\dot{s}$ on the geodesic $(b, d)$ with respect to the above two triangles is given by (see [1])

$$
\begin{aligned}
\dot{s}((b, d))= & \frac{V(c)-V(b)}{c-b}+\frac{V(d)-V(a)}{d-a}- \\
& \frac{V(b)-V(a)}{b-a}-\frac{V(d)-V(c)}{d-c} .
\end{aligned}
$$


Formula (27) is the first variation of the cross-ratio

$$
\operatorname{cr}(a, b, c, d)=\frac{(c-b)(d-a)}{(b-a)(d-c)}
$$

where $a, b, c, d \in \hat{\mathbf{R}}$ are given in the cyclic order on $\hat{\mathbf{R}}$. For this definition of the cross-ratio,

$$
\log c r(a, b, c, d)
$$

is the shear on the geodesic $(b, d)$ considered as a diagonal of the quadrilateral with vertices $a, b, c, d$.

Using the equation (27) and theorems 5.2 and 5.4 we immediately obtain

Corollary 6.1. Let $\dot{s}: \mathcal{F} \rightarrow \mathbf{R}$ be the shear function of a Zygmund bounded vector field on $\hat{\mathbf{R}}$. Then the infinitesimal shear function

$$
H(\dot{s}): \mathcal{F} \rightarrow \mathbf{R}
$$

of the vector field $H(V)$ obtained by taking the Hilbert transform of $V$ is given by (28)

$$
\begin{aligned}
H(\dot{s})((b, d))=\sum_{p \in \hat{Q} \sum_{n \in \mathbf{Z}} \dot{s}_{p}\left(e_{n}\right)\left[\frac{H\left(V_{e_{n}}\right)(c)-H\left(V_{e_{n}}\right)(b)}{c-b}+\frac{H\left(V_{e_{n}}\right)(d)-H\left(V_{e_{n}}\right)(a)}{d-a}\right.} \\
\left.-\frac{H\left(V_{e_{n}}\right)(b)-H\left(V_{e_{n}}\right)(a)}{b-a}-\frac{H\left(V_{e_{n}}\right)(d)-H\left(V_{e_{n}}\right)(c)}{d-c}\right]
\end{aligned}
$$

where $(b, d) \in \mathcal{F}$ is the common boundary side of the two complementary triangles of $\mathcal{F}$ with vertices $(a, b, d)$ and $(b, c, d)$, and $e_{n}$ is a geodesic of the fan $\mathcal{F}_{p}$ oriented such that $p$ is the initial point of $e_{n}$ for $n \geq 1$ and $p$ is the terminal point of $e_{n}$ for $n \leq 0$.

The above computation of the infinitesimal shear function $H(\dot{s})$ can be given in terms of the hyperbolic geometry of the underlining tesselation $\mathcal{F}$. Namely, let $(0, \infty)$ be the geodesic $e_{n}$ and let $(b, d)$ be the geodesic whose shear $H(\dot{s})((b, d))$ we want to compute. Let $a$ and $c$ be the other two endpoints of the ideal quadrilateral in $\mathbf{H} \backslash(\mathcal{F} \backslash\{(b, d)\})$.

Assume first that $a, b, c, d \notin\{0, \infty\}$ and let $V_{0, \infty}$ be given by (4). Then the contribution of $H\left(V_{0, \infty}\right)$ to $H(\dot{s})((b, d))$ is

$$
\frac{b}{c-b} \log \left|\frac{c}{b}\right|+\frac{d}{d-a} \log \left|\frac{d}{a}\right|-\frac{b}{b-a} \log \left|\frac{b}{a}\right|-\frac{d}{d-c} \log \left|\frac{d}{c}\right| .
$$

We write the above expression in terms of the cross-ratios as

$$
\begin{aligned}
& \quad c r(0, \infty, c, b) \log |\operatorname{cr}(0, b, \infty, c)|+\operatorname{cr}(0, \infty, a, d) \log |\operatorname{cr}(0, a, \infty, d)|- \\
& \quad \operatorname{cr}(0, \infty, a, b) \log |\operatorname{cr}(0, a, \infty, b)|-\operatorname{cr}(0, \infty, c, d) \log |\operatorname{cr}(0, b, \infty, c)| .
\end{aligned}
$$

Let $\delta_{a, d}$ be the hyperbolic distance between $(0, \infty)$ and $(a, d)$, and similarly for $\delta_{a, b}, \delta_{b, c}, \delta_{c, d}$. Then the expression (29) can be written as

$$
\begin{gathered}
\sinh ^{2}\left(\frac{\delta_{b, c}}{2}\right) \log \operatorname{coth}^{2}\left(\frac{\delta_{b, c}}{2}\right)+\cosh ^{2}\left(\frac{\delta_{a, d}}{2}\right) \log \operatorname{coth}^{2}\left(\frac{\delta_{a, d}}{2}\right)- \\
\cosh ^{2}\left(\frac{\delta_{a, b}}{2}\right) \log \operatorname{coth}^{2}\left(\frac{\delta_{a, b}}{2}\right)-\cosh ^{2}\left(\frac{\delta_{c, d}}{2}\right) \log \operatorname{coth}^{2}\left(\frac{\delta_{c, d}}{2}\right)
\end{gathered}
$$


If $a=0$ and $b, c, d \notin\{0, \infty\}$, then by similar reasoning we get that the contribution is

$$
\begin{array}{r}
\sinh ^{2}\left(\frac{\delta_{b, c}}{2}\right) \log \operatorname{coth}^{2}\left(\frac{\delta_{b, c}}{2}\right)+\log \operatorname{coth}^{2}\left(\frac{\delta_{b, d}}{2}\right)- \\
\cosh ^{2}\left(\frac{\delta_{c, d}}{2}\right) \log \operatorname{coth}^{2}\left(\frac{\delta_{c, d}}{2}\right) .
\end{array}
$$

If $d=\infty$ and $a, b, c \notin\{0, \infty\}$, then the contribution is

$$
\sinh ^{2}\left(\frac{\delta_{b, c}}{2}\right) \log \operatorname{coth}^{2}\left(\frac{\delta_{b, c}}{2}\right)-\cosh ^{2}\left(\frac{\delta_{a, b}}{2}\right) \log \operatorname{coth}^{2}\left(\frac{\delta_{a, b}}{2}\right) .
$$

If $a=0$ and $d=\infty$, then the contribution is

$$
\cosh ^{2}\left(\frac{\delta_{b, c}}{2}\right) \log \operatorname{coth}^{2}\left(\frac{\delta_{b, c}}{2}\right) .
$$

If $b=0$ and $d=\infty$, then the contribution is

$$
\log \tan ^{2}\left(\theta_{a, c}\right)
$$

where $\theta_{a, c}$ is the angle between geodesics $(0, \infty)$ and $(a, c)$.

Note that all the contributions to $H(\dot{s})((b, d))$ are expressed in terms of the invariants for the positions of the geodesics with endpoints $a, b, c, d, 0, \infty$ and they remain in force when the geodesic $(0, \infty)$ is replaced by an arbitrary geodesic. Thus we obtain

Corollary 6.2. Let $\dot{s}: \mathcal{F} \rightarrow \mathbf{R}$ be the infinitesimal shear function of a Zygmund bounded vector field $V$ on $\hat{\mathbf{R}}$. Then the infinitesimal shear function

$$
H(\dot{s}): \mathcal{F} \rightarrow \mathbf{R}
$$

of the vector field $H(V)$ obtained by taking the Hilbert transform of $V$ is given by

$$
H(\dot{s})((b, d))=\sum_{p \in \hat{Q}} \sum_{n \in \mathbf{Z}} \dot{s}\left(e_{n}\right) \Delta_{b, d}\left(e_{n}\right)
$$

where $(b, d) \in \mathcal{F}$ is the common boundary side of the two complementary triangles of $\mathcal{F}$ with vertices $(a, b, d)$ and $(b, c, d)$, and $e_{n}$ is a geodesic of the fan $\mathcal{F}_{p}$ oriented such that $p$ is the initial point of $e_{n}$ for $n \geq 1$ and $p$ is the terminal point of $e_{n}$ for $n \leq 0$, and $\Delta_{b, d}\left(e_{n}\right)$ is one of the expressions (30), (31), 32) (33), 34) depending on the relative positions of $(a, b, c, d)$ with respect to $e_{n}$.

\section{FOURIER COEFFICIENTS AND INFINITESIMAL SHEAR FUNCTIONS}

Let $V: S^{1} \rightarrow \mathbf{C}$ be a Zygmund bounded vector field on the unit circle of $S^{1}$. Let $\mathcal{F}$ be the Farey tesselation of the unit disk $\mathbf{D}$ which is obtained by taking the image of the Farey tesselation of $\mathbf{H}$ under the Möbius map $B: \mathbf{H} \rightarrow \mathbf{D}$ which maps 0,1 and $\infty$ onto $1, i$ and -1 . In this section, $V$ is normalized to vanish at $1, i$ and -1 . The infinitesimal shear function $\dot{s}: \mathcal{F} \rightarrow \mathbf{R}$ corresponding to $V$ satisfies the property (10) since all the considerations are geometric.

We express Fourier coefficients of a Zygmund bounded vector field $V$ on the unit circle $S^{1}$ in terms of its infinitesimal shear function defined on the Farey tesselation $\mathcal{F}$. Parametrize $S^{1}$ by associating to each point $z \in S^{1}$ its angle $\phi=\arg (z)$, where $\phi \in[0,2 \pi]$. Then, for $\phi_{0}<\phi_{1}$, the elementary shear vector field is defined by

$$
V_{\phi_{0}, \phi_{1}}(z)=\left\{\begin{array}{l}
\frac{\left(z-e^{i \phi_{0}}\right)\left(z-e^{i \phi_{1}}\right)}{e^{i \phi_{0}}-e^{i \phi_{1}}}, \text { for } \phi_{0}<\arg (z)<\phi_{1} \\
0, \quad \text { otherwise }
\end{array}\right.
$$


Given a fan $\mathcal{F}_{p}$ with the tip $p \in S^{1}$ of the Farey tesselation $\mathcal{F}$ in $\mathbf{D}$, denote by $V_{p}$ the vector field corresponding to a infinitesimal shear function which agrees with $\frac{1}{2} \dot{s}$ on $\mathcal{F}_{p}$ and which is zero on $\mathcal{F} \backslash \mathcal{F}_{p}$, and $V_{p}$ is normalized to be zero at $1, i$ and -1 . Namely,

$$
V_{p}(z)=\sum_{e_{n}=\left(e^{\left.i \phi_{0}^{n}, e^{i \phi_{1}^{n}}\right) \in \mathcal{F}_{p}}\right.} \frac{1}{2} \dot{s}\left(e_{n}\right) V_{\phi_{0}^{n}, \phi_{1}^{n}}(z) .
$$

Corollary 4.8 implies that

$$
V(z)=\sum_{p \in \mathcal{F}^{0}} V_{p}(z)
$$

where $\mathcal{F}^{0}$ is the set of all vertices of $\mathcal{F}$ and the convergence is absolute and uniform on $S^{1}$.

An elementary integration gives the $n$-th Fourier coefficient $\widehat{V}_{\phi_{0}, \phi_{1}}(n)$ of $V_{\phi_{0}, \phi_{1}}$ as

$$
\begin{array}{r}
\widehat{V}_{\phi_{0}, \phi_{1}}(n)=\frac{1}{2 \pi\left(e^{i \phi_{0}}-e^{i \phi_{1}}\right)}\left[\frac{e^{i(2-n) \phi_{1}}-e^{i(2-n) \phi_{0}}}{i(2-n)}-\left(e^{i \phi_{0}}+e^{i \phi_{1}}\right) \times\right. \\
\left.\quad \times \frac{e^{i(1-n) \phi_{1}}-e^{i(1-n) \phi_{0}}}{i(1-n)}+e^{i\left(\phi_{0}+\phi_{1}\right)} \frac{e^{-i n \phi_{1}}-e^{-i n \phi_{0}}}{-i n}\right]
\end{array}
$$

We have the following theorem.

Theorem 7.1. Let $V: S^{1} \rightarrow \mathbf{C}$ be a Zygmund vector field and let $\dot{s}: \mathcal{F} \rightarrow \mathbf{R}$ be the corresponding infinitesimal shear function. Then the $n$-th Fourier coefficient $\widehat{V}(n)$ of the vector field $V$ is given by

$$
\widehat{V}(n)=\sum_{p \in \mathcal{F}^{0}} \widehat{V}_{p}(n),
$$

where the convergence is absolute and

$$
\widehat{V}_{p}(n)=\sum_{e_{n}=\left(e^{\left.i \phi_{0}^{n}, e^{i \phi_{1}^{n}}\right) \in \mathcal{F}_{p}}\right.} \frac{1}{2} \dot{s}\left(e_{n}\right) \widehat{V}_{\phi_{0}^{n}, \phi_{1}^{n}}(n) .
$$

Proof. Since $\sum_{p \in \mathcal{F}^{0}} V_{p}(z)$ is converging uniformly on $S^{1}$ to $V(z)$, the first formula of the theorem is immediate. By Lemma 5.5 and the proof of Theorem 5.2, we have that $\left|V_{p}(z)\right|$ in a neighborhood of $p$ is increasing to infinity slower than the distance to $p$. This implies that the second formula of the theorem holds.

\section{The almost COMplex structure For Teichmüller SPACES of Finite SURFACES}

Let $S$ be a finite area hyperbolic surface with $s>0$ punctures. The hyperbolic plane $\mathbf{H}$ is the universal covering of $S$ such that the covering map $\pi: \mathbf{H} \rightarrow S$ is a local isometry. Let $G$ be the group of deck transformations and let $\tau$ be a locally finite ideal triangulation of $S$. Then $\tau$ lifts to a locally finite ideal triangulation $\tilde{\tau}$ of $\mathbf{H}$ which is invariant under $G$.

The Teichmüller space $T(S)$ of a Riemann surface $S$ is the homotopy class of all marked hyperbolic surfaces $f: S \rightarrow X$ up to post-composition by hyperbolic isometries. We denote the Teichmüller class of $f: S \rightarrow X$ by $[X, f]$. The Teichmüller space $T(S)$ maps into the space of functions from the set of geodesics $|\tau|$ of the triangulation $\tau$ into the real numbers $\mathbf{R}$ as follows. Let $c_{1}, \ldots, c_{s}$ be the set 
of punctures of $S$. Given a marked hyperbolic surface $f: S \rightarrow X$, we replace each curve in $|f(\tau)|$ with the geodesic of $X$ homotopic to it relative punctures. We obtain an ideal geodesic triangulation of the marked hyperbolic surface $X$ homotopic to $f(\tau)$. Then we assign to the marked hyperbolic surface $X$ the function from $|\tau|$ into $\mathbf{R}$ which maps each edge of $\tau$ to its shear with respect to $f(\tau)$. In this fashion, we obtain an injective map

$$
T(S) \rightarrow \mathbf{R}^{|\tau|}
$$

which is a real analytic diffeomorphism onto its image (see [30, 22]). The image of $T(S)$ consists of all functions $s:|\tau| \rightarrow \mathbf{R}$ such that for each puncture $c_{i}, i=1, \ldots, s$, we have

$$
\sum_{j=1}^{k(i)} s\left(e_{j}^{c_{i}}\right)=0
$$

where $e_{j}^{c_{i}}, j=1, \ldots, k(i)$, is the set of geodesics from $\tau$ having an ideal endpoint the cusp $c_{i}$ such that a single geodesic appears twice if and only if both of its endpoint are $c_{i}$.

A tangent vector to $T(S)$ at point $[X, f] \in T(S)$ is also described by a function in $\mathbf{R}^{|\tau|}$ as follows. Namely, a differentiable (in $t$ ) path of shear functions $s_{t}:|\tau| \rightarrow \mathbf{R}$, $t \in(-\epsilon, \epsilon)$, such that $s_{0}:|\tau| \rightarrow \mathbf{R}$ equals the shear function of $[X, f]$ describes a differentiable path in $T(S)$ through the point $[X, f]$. The derivative

$$
\left.\frac{d}{d t} s_{t}\right|_{t=0}=\dot{s}:|\tau| \rightarrow \mathbf{R}
$$

represents a tangent vector $v \in T_{[X, f]} T(S)$. In general, a function $\dot{s} \in \mathbf{R}^{|\tau|}$ represents a tangent vector at $T_{[X, f]} T(S)$ if and only if for each puncture $c_{i}$ it satisfies

$$
\sum_{j=1}^{k(i)} \dot{s}\left(e_{j}^{c_{i}}\right)=0
$$

where $e_{j}^{c_{i}}, j=1, \ldots, k(i)$, is the set of geodesics from $\tau$ having an ideal endpoint the cusp $c_{i}$ with possible repeating if both ends are at the same cusp as before.

Lift the triangulation $\tau$ of $S$ to a triangulation $\tilde{\tau}$ of $\mathbf{H}$. We normalize the covering map such that $\tilde{\tau}$ contains the geodesic $(0, \infty)$ and denote by $a_{-1}<0$ the endpoint of the geodesic in the fan of $\tilde{\tau}$ with tip $\infty$ adjacent to $(0, \infty)$ on the left. We choose the complementary triangle $\Delta_{0}$ with vertices $0, a_{-1}$ and $\infty$ to be the reference triangle for $\tilde{\tau}$ and orient all geodesics of $\tilde{\tau}$ to the left as seen from $\Delta_{0}$. Given a vertex $p \in \hat{\mathbf{R}}$ of $\tilde{\tau}$, let $\left\{e_{n}^{p}\right\}_{n \in \mathbf{Z}}$ be the set of edges of $\tilde{\tau}$ which have one endpoint $p$ such that $e_{n}^{p}$ is adjacent to $e_{n+1}^{p}$ for all $n \in \mathbf{Z}$, and that $e_{0}^{p}$ has the initial point $p$. The set $\left\{e_{n}^{p}\right\}_{n \in \mathbf{Z}}=\tilde{\tau}_{p}$ is said to be a fan of $\tilde{\tau}$ with tip $p$. Then, a map

$$
\tilde{s}:|\tilde{\tau}| \rightarrow \mathbf{R}
$$

represent a point in $T(S)$ if and only if $\tilde{s}$ is invariant under $G$ and for each vertex $p \in \tilde{\tau}_{0}$ we have

$$
\sum_{i=1}^{n(p)} \tilde{s}\left(e_{j+i}^{p}\right)=0
$$

where $e_{j+1}^{p}$ is identified with $e_{j+n(p)+1}^{p}$ by an element of $G$ and $j \in \mathbf{Z}$ is arbitrary. We choose $n(p)$ to be the smallest positive number such that $e_{j+1}^{p}$ is identified with 
$e_{j+n(p)+1}^{p}$ by an element of $G$. A function

$$
\dot{\tilde{s}}:|\tilde{\tau}| \rightarrow \mathbf{R}
$$

represent a tangent vector to a point in $T(S)$ if and only if $\dot{\tilde{s}}$ is invariant under $G$ and for each vertex $p \in \tilde{\tau}_{0}$ we have

$$
\sum_{i=1}^{n(p)} \dot{\tilde{s}}\left(e_{j+i}^{p}\right)=0
$$

where $e_{j+1}^{p}$ is identified with $e_{j+n(p)+1}^{p}$ by an element of $G$, and again we assume that $n(p)$ is the smallest such number. Note that $\dot{\tilde{s}}$ defines a tangent vector at an arbitrary point of $T(S)$.

Our goal in this section is to compute the Hilbert transform in terms of $\dot{\tilde{s}}:|\tilde{\tau}| \rightarrow$ R. Let $\Delta_{0}$ be the reference complementary triangle to $\tilde{\tau}$ whose ideal vertices are 0 , $a_{1}$ and $\infty$. Let $\left\{e_{n}^{\infty}\right\}_{n \in \mathbf{Z}}$ be the fan of $\tilde{\tau}$ with tip $\infty$ such that $e_{0}$ has initial point $\infty$ and terminal point $0, e_{1}$ is to the left of $e_{0}$ and $e_{n}$ is adjacent to $e_{n+1}$ for all $n \in \mathbf{Z}$. Let $a_{n}$ be the endpoint of $e_{n}$ different from $\infty$. Let $V_{\infty}$ be the vector field normalized to be zero at $0, a_{1}$ and $\infty$ whose shear function agree with $\dot{\tilde{s}}$ on $\tilde{\tau}_{\infty}$ and equals zero on all other geodesics of $\tilde{\tau}$. The lemma below proves that $V_{\infty}$ is a Zygmund vector field.

Lemma 8.1. Let $n \geq 1$ be a fixed integer and let $\dot{\tilde{s}}: \tilde{\tau}_{\infty} \rightarrow \mathbf{R}$ be a function such that

and

$$
\dot{\tilde{s}}\left(e_{j}^{\infty}\right)=\dot{\tilde{s}}\left(e_{j+n}^{\infty}\right)
$$

for all $j \in \mathbf{Z}$. Let $\dot{\tilde{s}}_{N}: \tilde{\tau}_{\infty} \rightarrow \mathbf{R}$ be defined by $\dot{\tilde{s}}_{N}\left(e_{i}^{\infty}\right)=\dot{\tilde{s}}\left(e_{i}^{\infty}\right)$ if $-N n \leq i \leq N n-1$, and $\dot{\tilde{s}}_{N}\left(e_{i}^{\infty}\right)=0$ otherwise. Let $V_{\infty}^{N}$ be the vector field corresponding to $\dot{\tilde{s}}_{N}$ and let $V_{\infty}$ be the vector field corresponding to $\dot{\tilde{s}}$. Then $V_{\infty}$ and $V_{\infty}^{N}$ are Zygmund vector fields whose Zygmund constants are bounded by some $C_{3}>0$ for all $N \in \mathbf{N}$.

Proof. Let $g(x)=x+k$, for $k>0$, be a generator of the subgroup of $G$ fixing $\infty$. Then we have $a_{i}+k=a_{i+n}$ for all $i \in \mathbf{Z}$ by the invariance of $\tilde{\tau}$ under $G$. We normalize $V_{\infty}$ and $V_{\infty}^{N}$ to be zero on the interval $\left[a_{-1}, a_{0}=0\right]$. By (37), the contribution of

$$
\dot{\tilde{s}}\left(e_{0}^{\infty}\right)\left(x-a_{0}\right)+\dot{\tilde{s}}\left(e_{1}^{\infty}\right)\left(x-a_{1}\right)+\cdots+\dot{\tilde{s}}\left(e_{n-1}^{\infty}\right)\left(x-a_{n-1}\right)
$$

is equal to

$$
-\sum_{i=0}^{n-1} \dot{\tilde{s}}\left(e_{i}^{\infty}\right) a_{i}
$$

Moreover, for $j>0$ and $j \equiv 0 \bmod n$, we have

$$
\sum_{i=0}^{n-1} \dot{\tilde{s}}\left(e_{j+i}^{\infty}\right)\left(x-a_{j+i}\right)=\sum_{i=0}^{n-1} \dot{\tilde{s}}\left(e_{i}^{\infty}\right)\left(x-\frac{j}{n} k-a_{i}\right)=-\sum_{i=0}^{n-1} \dot{\tilde{s}}\left(e_{i}^{\infty}\right) a_{i}
$$

because $\dot{\tilde{s}}\left(e_{j+i}^{\infty}\right)=\dot{\tilde{s}}\left(e_{i}^{\infty}\right)$ and $a_{j+i}=a_{i}+\frac{j}{n} k$ by the invariance of $\dot{\tilde{s}}$ and $\tilde{\tau}$ under $G$. 
If $j<0$ and $j \equiv 0 \bmod n$, then, in a similar fashion, we obtain

$$
\sum_{i=0}^{n-1} \dot{\tilde{s}}\left(e_{j-1-i}^{\infty}\right)\left(x-a_{j-1-i}\right)=-\sum_{i=0}^{n-1} \dot{\tilde{s}}\left(e_{-i-1}^{\infty}\right) a_{-i-1} .
$$

Define

$C=\max _{0<i_{0}<n}\left\{\sup _{x \in\left[a_{i_{0}}, a_{i_{0}+1}\right]}\left|\sum_{i=0}^{i_{0}} \dot{\tilde{s}}\left(e_{i}^{\infty}\right)\left(x-a_{i}\right)\right|+\sup _{x \in\left[a_{-i_{0}-1}, a_{-i_{0}}\right]}\left|\sum_{i=1}^{i_{0}} \dot{\tilde{s}}\left(e_{-i}^{\infty}\right)\left(x-a_{-i}\right)\right|\right\}$

The above estimates give that

$$
\left|V_{\infty}(x)\right| \leq C+\frac{|x|}{k} C_{1}
$$

and

$$
\left|V_{\infty}^{N}(x)\right| \leq C+C_{1} N
$$

for all $x \in \mathbf{R}$, where $C$ is defined above and

$$
C_{1}=\max \left\{\left|\sum_{i=0}^{n-1} \dot{\tilde{s}}\left(e_{i}^{\infty}\right) a_{i}\right|,\left|\sum_{i=0}^{n-1} \dot{\tilde{s}}\left(e_{-i-1}^{\infty}\right) a_{-i-1}\right|\right\} .
$$

We show that $V_{\infty}(x)$ and $V_{\infty}^{N}(x)$ are Lipschitz continuous with the constants bounded independently of $N$. Let $x \in \mathbf{R}$ and $t>0$. By adding a linear term and by the invariance of $V_{\infty}(x)$ under the action of $g(x)=x+k$, we assume that $0 \leq x \leq k$.

If $t \leq k$, then an easy argument shows that

$$
\left|V_{\infty}^{N}(x+t)-V_{\infty}^{N}(x)\right|,\left|V_{\infty}(x+t)-V_{\infty}(x)\right| \leq C_{2} t
$$

and

$$
\left|V_{\infty}^{N}(x)-V_{\infty}^{N}(x-t)\right|,\left|V_{\infty}(x)-V_{\infty}(x-t)\right| \leq C_{2} t,
$$

where $C_{2}=\max \left\{1, \sum_{i=0}^{n-1}\left|\dot{\tilde{s}}\left(e_{i}^{\infty}\right)\right|\right\}$.

Assume that $t>k$. Then (40) and (41) imply that

$$
\left|V_{\infty}^{N}(x+t)-V_{\infty}^{N}(x)\right|,\left|V_{\infty}(x+t)-V_{\infty}(x)\right| \leq 2 \frac{C_{1}+C_{2}}{k} t
$$

and

$$
\left|V_{\infty}^{N}(x)-V_{\infty}^{N}(x-t)\right|,\left|V_{\infty}(x)-V_{\infty}(x-t)\right| \leq 2 \frac{C_{1}+C_{2}}{k} t,
$$

where $C_{1}, C_{2}$ are as above. This implies that $V_{\infty}$ and $V_{\infty}^{N}$ are Zygmund bounded with bound $C_{3}=4\left(\frac{C_{1}+C_{2}}{k}+C_{2}\right)$.

Since $V_{\infty}$ and $V_{\infty}^{N}$ have their Zygmund norms bounded independently of $N$, it follows that their cross-ratio norms are uniformly bounded as well. Let $\tilde{\tau}^{0}$ be the set of vertices of $\tilde{\tau}$. This implies that $V_{p}$ and $V_{p}^{N}$ are Zygmund bounded with Zygmund bounds independent of $N$ for all $p \in \tilde{\tau}^{0}$, where $V_{p}^{N}, V_{p}$ are defined analogous to $V_{\infty}^{N}, V_{\infty}$.

Theorem 8.2. Let $S$ be a finite area punctured hyperbolic Riemann surface equipped with an ideal geodesic triangulation $\tau$. Let $\tilde{\tau}=\left\{e_{n}\right\}_{n \in \mathbf{N}}$ be the lift to the hyperbolic plane $\mathbf{H}$ of $\tau$ and let $\left\{V_{e_{n}}\right\}_{n \in \mathbf{N}}$ be the elementary vector fields corresponding to the 
geodesics $e_{n}$ of $\tilde{\tau}$. Let $V(x)$ be a Zygmund vector field on $\hat{\mathbf{R}}$ representing an infinitesimal deformation of $S$ and let $\dot{\tilde{s}}: \tilde{\tau} \rightarrow \mathbf{R}$ be the corresponding shear function. Then the series

$$
\sum_{n \in \mathbf{N}} \dot{\tilde{s}}\left(e_{n}\right) V_{e_{n}}(x)
$$

converges to $V(x)$ uniformly on compact subsets of $\mathbf{R}$.

Proof. We follow the idea in the proof of Theorem 4.1. Let $\left\{p_{i}\right\}_{i \in \hat{\mathbf{Q}}}$ be the set of vertices of $\tilde{\tau}$. There exists $\delta>0$, such that for any two fans $\tilde{\tau}_{p_{1}}$ and $\tilde{\tau}_{p_{2}}$ which do not have a common geodesic the minimum distance between the geodesics of $\tilde{\tau}_{p_{1}}$ and $\tilde{\tau}_{p_{2}}$ is at least $\delta$. This is a direct consequence of the invariance of $\tilde{\tau}$ under a co-finite group $G$.

Let $I \subset \mathbf{R}$ be a closed, bounded interval in $\mathbf{R}$. For $x \in I$, let $r_{x}$ denote a geodesic ray that connects $i \in \mathbf{H}$ to $x \in I \subset \mathbf{R}$. Given $\epsilon>0$, there exists a finite set of tips $\left\{p_{1}, \ldots, p_{s}\right\} \subset \tilde{\tau}^{0}, s=s(\epsilon)$, such that if a geodesic of the fan with a tip $p \in \tilde{\tau}^{0}$ intersects $r_{x}$ for some $x \in I$ then either all geodesics in $\tilde{\tau}_{p}$ have their Euclidean lengths less than $\epsilon$, or $p \in\left\{p_{1}, \ldots, p_{s}\right\}$ and all fans whose geodesics intersect $r_{x}$ before the geodesics of $\tilde{\tau}_{p}$ are also in $\left\{p_{1}, \ldots, p_{s}\right\}$. Let $n\left(p_{i}\right)$ stand for the number of elements in $\tilde{\tau}_{p_{i}}$ that form a fundamental set for the action by the subgroup of $G$ that stabilizes $p_{i}$. There exists $N=N(\epsilon, s) \in \mathbf{N}$ such that all geodesics $e_{j}^{p_{i}}$ of $\tilde{\tau}_{p_{i}}$, $i=1, \ldots, s$, for either $j>N n\left(p_{i}\right)$ or $j<-N n\left(p_{i}\right)$ have their Euclidean sizes less that $\epsilon$.

By our construction, the sum

$$
\sum_{i=1}^{s} V_{p_{i}}^{N}(x)
$$

agrees with $V(x)$ on an $\epsilon$-net for $I$. Since the Zygmund norms of each $V_{p}^{N}$ are uniformly bounded for $p \in \tilde{\tau}^{0}$ and $N \in \mathbf{N}$ and since the ideal triangulation has positive minimum distance between geodesics of two disjoint fans, it follows that the above sum converges uniformly on $I$ to $V(x)$ as $\epsilon \rightarrow 0$ similar to proof of Theorem 4.1. To finish the proof, it is enough to note that each $V_{p}^{N}$ is a finite sum of elementary shear vector fields $V_{e}$ for $e \in \tilde{\tau}$ with the coefficients $\dot{\tilde{s}}(e)$.

We use the above theorem to establish a formula for the Hilbert transform in terms of infinitesimal shear function of Zygmund vector field.

Theorem 8.3. Let $V: \mathbf{R} \rightarrow \mathbf{R}$ be a Zygmund vector field which is invariant under a co-finite group $G$ and let $\tilde{\tau}$ be an ideal triangulation of $\mathbf{H}$ invariant under $G$. Let $\dot{\tilde{s}}: \tilde{\tau} \rightarrow \mathbf{R}$ be the infinitesimal shear function of $V$. Then the Hilbert transform $H(V)$ is given by

$$
H(V)(x)=\sum_{n \in \mathbf{N}} \dot{\tilde{s}}\left(e_{n}\right) H\left(V_{e_{n}}\right)(x)
$$

where $\left\{e_{n}\right\}_{n \in \mathbf{N}}$ are the geodesics of $\tilde{\tau}$ given in a sequence and $H\left(V_{e_{n}}\right)$ is defined by (8).

Proof. We established that the quantity

$$
\sum_{i=1}^{s} V_{p_{i}}^{N}(x)
$$


in the proof of Theorem 8.2 converges uniformly on compact subsets of $\mathbf{R}$ and that each $V_{p_{i}}^{N}$ is Zygmund bounded with uniformly bounded Zygmund norms. Then Proposition 4.7 implies that $\sum_{i=1}^{s} V_{p_{i}}^{N}(x)$ have uniformly bounded Zygmund norms. Then Lemma 5.1 implies that

$$
H\left(\sum_{i=1}^{s} V_{p_{i}}^{N}(x)\right)=\sum_{i=1}^{s} H\left(V_{p_{i}}^{N}\right)(x) \rightarrow H(V)(x)
$$

as $\epsilon \rightarrow 0$. The theorem follows because each $V_{p_{i}}^{N}$ is a fite linear combination of simple shear vector fields.

We also obtain an expression of the infinitesimal shear function for the Hilbert transform similarly to the case when Zygmund vector field is not invariant.

Corollary 8.4. Let $\dot{\tilde{s}}: \tilde{\tau} \rightarrow \mathbf{R}$ be the infinitesimal shear function of a Zygmund vector field $V$ invariant under a co-finite group $G$, where $\tilde{\tau}$ is an ideal triangulation of $\mathbf{H}$ invariant under $G$. Then the infinitesimal shear function

$$
H(\dot{\tilde{s}}): \tilde{\tau} \rightarrow \mathbf{R}
$$

of the vector field $H(V)$ obtained by taking the Hilbert transform of $V$ is given by

$$
H(\dot{\tilde{s}})((b, d))=\sum_{n \in \mathbf{N}} \dot{\tilde{s}}\left(e_{n}\right) \Delta_{b, d}\left(e_{n}\right)
$$

where $(b, d) \in \mathcal{F}$ is the common boundary side of the two complementary triangles of $\mathcal{F}$ with vertices $(a, b, d)$ and $(b, c, d), \tilde{\tau}=\left\{e_{n}\right\}_{n \in \mathbf{N}}$, and $\Delta_{b, d}\left(e_{n}\right)$ is one of the expressions (30), (31), (32), (33), 34) depending on the relative positions of $(a, b, c, d)$ with respect to $e_{n}$.

\section{The Weil-Petersson metric for Teichmüller spaces of finite SURFACES IN SHEAR COORDINATES}

We described above the Hilbert transform on the tangent vectors to the Teichmüller space $T(S)$ of a finite area hyperbolic surface $S$ with $s>0$ punctures. The Hilbert transform is the almost complex structure to $T(S)$ (see [20]). The Weil-Petersson metric $g_{W P}$ is Kähler [4, 33, 34. The symplectic two form of the Weil-Petersson metric in the shear coordinates is simply given as twice the Thurston algebraic intersection form on the weights induced by the tangent vectors on the train track associated to the ideal triangulation $\tau$ of $S$ ([8], see also [33, [23]). Therefore, we obtain the following formula in terms of shear coordinates.

Theorem 9.1. Let $S$ be a finite area hyperbolic surface with $s>0$ punctures and let $\tau$ be an ideal triangulation of $S$. Given two infinitesimal shear functions

$$
\dot{s}_{1}, \dot{s}_{2}: \tilde{\tau} \rightarrow \mathbf{R}
$$

which represent two tangent vectors $v_{1}, v_{2}$ to the Teichmüller space $T(S)$ of $S$ at the basepoint $[S, i d]$, the Weil-Petersson inner product is given by

$$
g_{W P}\left(v_{1}, v_{2}\right)=2 i\left(\dot{s}_{1}, H\left(\dot{s}_{2}\right)\right)
$$

where $H\left(\dot{s}_{2}\right)$ is the infinitesimal shear function of the Hilbert transform $H\left(v_{2}\right)$ and $i(\cdot, \cdot)$ is the Thurston's algebraic intersection number between infinitesimal shear functions defined on $\tau$. 


\section{Continuous extension of the Hilbert transform and the}

Weil-Petersson metric to The BOUndary of the Teichmüller space

Let $S$ be a finite area hyperbolic surface with $s>0$ punctures and let $\bar{T}(S)$ be the augmented Teichmüller space of $S$ [1], 5], 35]. Our goal is to show that the Hilbert transform and the Weil-Petersson metric extend by continuity to the completion of $T(S)$. Masur [18] proved that the Weil-Petersson hermitian metric continuously extend to the augmented Teichmüller space $\bar{T}(S)$. More recently, Roger 27] showed that the Weil-Petersson symplectic two form on $T(S)$ extends by continuity to the augmented Teichmüller space $\bar{T}(S)$ using the shear parametrization of $T(S)$ with appropriate degeneration of the ideal triangulation when the point is in $\bar{T}(S) \backslash T(S)$. We show that the Hilbert transform in terms of the shears for $T(S)$ extends by continuity to the augmented Teichmüller space $\bar{T}(S)$.

One description of the manifold structure on the augmented Teichmüller space $\bar{T}(S)$ is given in terms of the plumbing coordinates for a neighborhood of a point on the boundary of $T(S)$ [1, 5]. Another description is given by extending the Fenchel-Nielsen coordinates for $T(S)$ to allow the lengths of some of the closed geodesics of the pants decomposition to be zero [1], [5], 35]. Let $\sigma=\left\{\gamma_{1}, \ldots, \gamma_{k}\right\}$ be a set of mutually non-homotopic, non-intersecting and non-trivial simple closed curves on $S$. We denote by $\mathcal{S}(\sigma)$ the set of all (marked) nodded Riemann surfaces $R$ obtained from $S$ by pinching curves in $\sigma$. Then the augmented Teichmüller space consists of $T(S)$ and $\mathcal{S}(\sigma)$ over all $\sigma$ as above.

We use the plumbing coordinates for our purposes. Let $R_{t}, 0 \leq t<1$, be a path of marked Riemann surfaces in $T(S)$ such that its limit point $R^{*}$ as $t \rightarrow 1$ is on the boundary of $T(S)$ in $\mathcal{S}(\sigma)$. Therefore $R^{*}$ is a nodded Riemann surface with nodes corresponding to the curves of $\sigma$. The path $R_{t}$ is described by the plumbing construction in the neighborhood of the nodes in $R^{*}$ (see [18]). A tangent vector to $R^{*}$ on the boundary $\bar{T}(S) \backslash T(S)$ is represented by a (smooth) Beltrami differential $\nu$ which is supported on $R^{*} \backslash U$, where $U$ is the union of neighborhoods of nodes of $R^{*}$ which correspond to the curves in $\sigma$. Then the Beltrami differential $\nu$ on $R_{t} \backslash U$ represents tangent vectors at $R_{t} \in T(S)$ which converge to $\nu$ on $R^{*}$. We normalize the universal coverings $\pi_{t}: \mathbf{H} \rightarrow R_{t}$ and $\pi: \mathbf{H} \rightarrow R^{*}$ such that they map $i \in \mathbf{H}$ onto a fixed point $p \in R_{t} \backslash U=R^{*} \backslash U$. Since the differentiable structures on $R_{t} \backslash U$ and $R^{*} \backslash U$ are the same, we can further normalize $\pi_{t}, \pi$ such that they map a fixed tangent direction at $i \in \mathbf{H}$ onto a fixed tangent direction at $p$.

Let $\tilde{\nu}_{t}=\left(\pi_{t}\right)_{*} \nu$ and $\tilde{\nu}=(\pi)_{*} \nu$ be the pull-backs of $\nu$ by the universal coverings. The Zygmund vector fields $V_{t}$ on $\hat{\mathbf{R}}$ corresponding to $\tilde{\nu}_{t}$ converge pointwise on $\hat{\mathbf{R}}$ to the Zygmund vector field $V$ corresponding to $\tilde{\nu}$ because $\tilde{\nu}_{t}$ converges pointwise to $\tilde{\nu}$ on $\mathbf{H}$. The Zygmund norms of $V_{t}, V$ are uniformly bounded because $\left\|\tilde{\nu}_{t}\right\|_{\infty},\|\tilde{\nu}\|_{\infty}$ are uniformly bounded.

We describe the convergence of tangent vectors as they approach to the boundary of $T(S)$ in terms of shear parametrization. We first recall the convergence of the path of infinitesimal shear functions corresponding to a path of points in $T(S)$ that converges to a boundary point $R \in \mathcal{S}(\sigma)$ described by J. Roger [27]. Let $\lambda=\left\{h_{1}, h_{2}, \ldots, h_{n}\right\}$ be an ideal triangulation of the punctured surface $S$. Consider the arcs $h_{i} \cap(S \backslash \sigma)$ for $i=1,2, \ldots, n$. They divide the surface $S \backslash \sigma$ into triangles and bigons [27]. We group the edges of $\lambda \cap(S \backslash \sigma)$ into homotopy classes relative to punctures of $S$ and to $\sigma$. In this fashion an ideal triangulation $\mu=\left\{g_{1}, g_{2}, \ldots, g_{n}\right\}$ 
of $S \backslash \sigma$ is obtained. Let $k_{i, j}$ be the number of arcs of $h_{j} \cap(S \backslash \sigma)$ that are homotopic to $g_{i}$. Then

Proposition 10.1. 27. Let $R_{t} \in T(S)$ for $t \in[0,1)$ be a path which converges to $R^{*} \in \mathcal{S}(\sigma) \subset \bar{T}(S)$ as $t \rightarrow 1$. Let $s_{t}: \lambda \rightarrow \mathbf{R}$ be the infinitesimal shear function for $R_{t}$ and let $s^{*}: \mu \rightarrow \mathbf{R}$ be the infinitesimal shear function for $R^{*}$. Then

$$
\lim _{t \rightarrow 1} \sum_{j=1}^{n} k_{i, j} s_{t}\left(h_{j}\right)=s^{*}\left(g_{i}\right) .
$$

In other words, the value $s^{*}\left(g_{i}\right)$ is the limit of the sum of the values of $s_{t}$ on the arcs of $\lambda \cap(S \cap \sigma)$ which are homotopic to $g_{i}$.

We continue to work with the path $R_{t} \in T(S)$ for $t \in[0,1)$ which converges to $R^{*} \in \mathcal{S}(\sigma)$ as $t \rightarrow 1$. Let $u_{t}$ and $u^{*}$ be tangent vectors based at $R_{t}$ and $R^{*}$ such that $R_{t} \rightarrow R^{*}$ and $u_{t} \rightarrow u^{*}$ as $t \rightarrow 1$. Let $s_{t}, \dot{s}_{t}: \lambda \rightarrow \mathbf{R}$ and $s^{*}, \dot{s}^{*}: \mu \rightarrow \mathbf{R}$ be the infinitesimal shear functions for $R_{t}, u_{t}, R^{*}$ and $u^{*}$, respectively. Then the sum of $s_{t}, \dot{s}_{t}$ shears for the geodesics in $\lambda$ at each puncture of $S$ is zero. Similarly, the sum of $s^{*}, \dot{s}^{*}$ shears for the geodesics in $\mu$ at each puncture of $S \backslash \sigma$ is zero, where $\alpha \in \sigma$ is considered a puncture of $S \backslash \sigma$.

Proposition 10.2. Assume that $s_{t}, \dot{s}_{t}, s^{*}, \dot{s}^{*}$ are as above. Then

$$
\lim _{t \rightarrow 1} \sum_{j=1}^{n} k_{i, j} \dot{s}_{t}\left(h_{j}\right)=\dot{s}^{*}\left(g_{i}\right) .
$$

Proof. Let $R_{t}^{k}$ be the component of $R_{t} \backslash \sigma$ which corresponds to the component of $S \backslash \sigma$ which contains arcs homotopic to $g_{i}$. Let $p \in R_{t}^{k} \backslash U=R^{*} \backslash U$ be a fixed point with a fixed tangent direction. Let $\pi_{t}: \mathbf{H} \rightarrow R_{t}$ be the universal covering of $R_{t}$ chosen such that $\pi_{t}(i)=p$ and a fixed tangent direction at $i \in \mathbf{H}$ is mapped onto the fixed tangent direction at $p \in R_{t}^{k}$. Let $V_{t}$ and $V^{*}$ be the Zygmund vector fields corresponding to the tangent vectors $u_{t}$ and $u^{*}$. By the above, we have that $V_{t}$ converges to $V^{*}$ pointwise on $\hat{\mathbf{R}}$.

The normalization of the universal covering $\pi_{t}: \mathbf{H} \rightarrow R_{t}$ implies that the lifts $\left(\pi_{t}\right)^{-1}(\sigma)$ leave every compact subset of $\mathbf{H}$. It follows that $\left(\pi_{t}\right)^{-1}(\lambda)$ converges to $\pi^{-1}(\mu)$.

Let $\tilde{g}_{i}$ be a lift of $g_{i}$ to $\mathbf{H}$ under the covering map $\pi$. Recall that $g_{i}$ corresponds to a homotopy class of arcs in $\lambda \cap(S \backslash \sigma)$. If $g_{i}$ does not end at $\sigma$, then there is a unique $h_{i} \in \mu$ which corresponds to it. Let $\tilde{h}_{i}(t)$ be the lift of $h_{i}$ under $\pi_{t}$ such that $\tilde{h}_{i}(t) \rightarrow \tilde{g}_{i}$ as $t \rightarrow 1$. If $g_{i}$ has one or two ends at $\sigma$, then there exists finitely many geodesics $h_{i, 1}, \ldots, h_{i, j} \in \lambda$ written with multiplicity such that $h_{i, l} \cap(S \backslash \sigma)$ for $l=1, \ldots, j$ is homotopic to $g_{i}$. Let $\tilde{h}_{i, l}(t)$ be the lift of $h_{i, l}$ under $\pi_{t}$ for $l=1, \ldots, j$ such that $\tilde{h}_{i, l}(t)$ converges to $\tilde{g}_{i}$ as $t \rightarrow 1$.

We can assume that $\tilde{h}_{i, 1}(t), \tilde{h}_{i, 2}(t) \ldots, \tilde{h}_{i, j}(t)$ are given in order such that each $\tilde{h}_{i, l}$ is in between $\tilde{h}_{i, l-1}$ and $\tilde{h}_{i, l+1}$ for $l=2, \ldots, j-1$. Then $\tilde{h}_{i, l}$ and $\tilde{h}_{i, l+1}$ share a common endpoint.

Let $a, b, c, d \in \hat{\mathbf{R}}$ be the endpoints of a quadrilateral with the diagonal $(b, d)$. Then the shear $\dot{s}$ of the diagonal $(b, d)$ associated to a vector field $V$ on $\hat{\mathbf{R}}$ is given by

$$
\dot{s}((b, d))=\frac{V(c)-V(b)}{c-b}+\frac{V(d)-V(a)}{d-a}-\frac{V(d)-V(c)}{d-c}-\frac{V(b)-V(a)}{b-a} .
$$


We write $V(c, b)$ instead of $\frac{V(c)-V(b)}{c-b}$ for short and similar for the other expressions. Note that the expression $\dot{s}((b, d))$ is the sum of four terms with positive or negative sign in front. In fact, the sign in front can be determined as follows. Orient the boundary sides of the triangle $T$ with vertices $(a, b, d)$ such that $T$ stays on the left of the boundary sides. If the terminal endpoint of a side $t_{1}$ of $T$ coincides with the initial point of a side $t_{2}$ of $T$ then we say that $t_{1}$ comes before $t_{2}$, otherwise $t_{1}$ comes after $t_{2}$. The quadruple $(a, b, c, d)$ divides $\hat{\mathbf{R}}$ into four intervals. Each expression above corresponds to one of the four intervals. The sign in front of the expression is positive if the geodesic whose endpoints agree with the endpoints of the interval comes after the diagonal $(b, d)$, and it is negative otherwise.

Each geodesic of the sequence $\tilde{h}_{i, 1}(t), \tilde{h}_{i, 2}(t) \ldots, \tilde{h}_{i, j}(t)$ intersects one lift $\tilde{\alpha}_{t}$ of some $\alpha \in \sigma$ under the covering map $\pi_{t}$. Thus $\tilde{\alpha}_{t}$ separates one endpoint of each geodesic of the sequence from $i \in \mathbf{H}$ when $t$ is close enough to 1 . The set of other endpoints is either separated from $i \in \mathbf{H}$ by another lift $\tilde{\alpha}^{\prime}$ of a geodesic $\alpha^{\prime} \in \sigma$, or all endpoints are equal. In both cases, the sequence of geodesics $\tilde{h}_{i, 1}(t), \tilde{h}_{i, 2}(t) \ldots, \tilde{h}_{i, j}(t)$ converges to $\tilde{g}_{i}$ as $t \rightarrow 1$. Each two consecutive geodesics $\tilde{h}_{i, l}(t), \tilde{h}_{i, l+1}(t)$ form a hyperbolic wedge and we make a hyperbolic triangle by adding a geodesic to this wedge whose endpoints are the endpoints of the two geodesic which are not common. The added geodesic is separated from $i \in \mathbf{H}$ by either $\tilde{\alpha}$ or $\tilde{\alpha}^{\prime}$. These added geodesic are small as $t \rightarrow 1$ in the Euclidean metric on $\mathbf{H} \cup \mathbf{R}$. We add a triangle $T_{1}$ to the side of $\tilde{h}_{i, 1}$ opposite $\tilde{h}_{i, 2}$ and a triangle $T_{2}$ to the side of $\tilde{h}_{i, j}$ opposite $\tilde{h}_{i, j-1}$. The added triangles $T_{1}$ and $T_{2}$ have all their sides in $\tilde{\lambda}$. We obtained a sequence of adjacent triangles such that the union of each two adjacent triangles forms a quadrilateral whose diagonal is in the sequence $\tilde{h}_{i, 1}(t), \tilde{h}_{i, 2}(t) \ldots, \tilde{h}_{i, j}(t)$. The added geodesics are small as $t \rightarrow 1$ and each of them appears as a boundary side of exactly two quadrilaterals which share a triangle.

We consider the sum $\sum_{l=1}^{j} \dot{\tilde{s}}_{t}\left(h_{i, l}(t)\right)$, where $\dot{\tilde{s}}_{t}\left(\tilde{h}_{i, l}\right)=V_{t}\left(d_{l}, a_{l}\right)+V_{t}\left(c_{l}, b_{l}\right)-$ $V_{t}\left(d_{l}, c_{l}\right)-V_{t}\left(b_{l}, a_{l}\right)$ as before and $\left(a_{l}, b_{l}, c_{l}, d_{l}\right)$ are the vertices of the quadrilateral whose diagonal is $\tilde{h}_{i, l}(t)$. Each small added geodesic $\left(a^{\prime}, b^{\prime}\right)$ is contained in exactly two adjacent quadrilaterals. The sign of $V\left(a^{\prime}, b^{\prime}\right)$ is determined with respect to the diagonal of the quadrilateral. Since two quadrilaterals have different diagonals it follows that the two terms for $V_{t}\left(a^{\prime}, b^{\prime}\right)$ have different signs in the sum and they cancel out.

We continue the analysis of the terms in the above sum. Note that $\tilde{h}_{i, 1}(t)$ and $\tilde{h}_{i, j}(t)$ appear as boundary sides of exactly one quadrilateral and that $\tilde{h}_{i, l}(t)$ for $l=2, \ldots, j-1$ appear as boundary sides of exactly two quadrilaterals. Let $\tilde{h}_{i, l}(t)=\left(b_{l}(t), d_{l}(t)\right), \tilde{h}_{i, l+1}(t)\left(b_{l+1}(t), d_{l+1}(t)\right)$ be two adjacent geodesics in the above sequence which are boundary sides of the above triangle with one small side, where either $b_{l}(t)=b_{l+1}(t)$ and $\left.\left.d_{l}(t)\right) \neq d_{l+1}(t)\right)$, or $b_{l}(t) \neq b_{l+1}(t)$ and $\left.\left.d_{l}(t)\right)=d_{l+1}(t)\right)$. The triangle appears as one half of two quadrilaterals which implies that $V_{t}\left(b_{l}(t), d_{l}(t)\right)$ and $V_{t}\left(b_{l+1}(t), d_{l+1}(t)\right)$ have different signs in the above sum. It is clear that $\left|V_{t}\left(b_{l}(t), d_{l}(t)\right)-V_{t}\left(b_{l+1}(t), d_{l+1}(t)\right)\right| \rightarrow 0$ as $t \rightarrow 1$ because $b_{l}(t)$ and $b_{l+1}(t)$, and $d_{l}(t)$ and $d_{l+1}(t)$ are close for $t$ close to 1 . It follows that the total sum of terms $V_{t}\left(b_{l}(t), d_{l}(t)\right)$ for the sequence $\left\{\tilde{h}_{i, l}(t)\right\}_{l=1}^{j}$ converges to zero as $t \rightarrow 1$.

Let $a(t)$ be the third vertex of $T_{1}$ and let $c(t)$ be the third vertex of $T_{2}$. Then $a(t)$ and $c(t)$ converge as $t \rightarrow 1$ to the vertices of the quadrilateral which is the 
union of two adjacent triangles in $\mathbf{H} \backslash \pi^{-1}(\mu)$ and which has $\tilde{g}_{i}$ as its diagonal. The above considerations imply that

$$
\begin{array}{r}
\lim _{t \rightarrow 0} \sum_{l=1}^{j} \dot{s}_{t}\left(h_{i, l}\right)=\lim _{t \rightarrow 0}\left[V_{t}\left(c(t), b_{i, j}(t)\right)+V_{t}\left(d_{i, 1}(t), a(t)\right)-\right. \\
\left.V_{t}\left(b_{i, 1}(t), a(t)\right)-V_{t}\left(d_{i, j}(t), c(t)\right)\right]=V(c, b)+ \\
V(d, a)-V(b, a)-V(d, c)=\dot{s}^{*}\left(g_{i}\right)
\end{array}
$$

where $g_{i}=(b, d)$. The proposition follows because $\sum_{l=1}^{j} \dot{s}_{t}\left(h_{i, l}\right)=\sum_{j=1}^{n} k_{i, j} \dot{s}_{t}\left(h_{j}\right)$ by the definition of $k_{i, j}$.

Let $u_{t}$ for $t \in[0,1)$ be a path of tangent vectors to $T(S)$ that limits to a tangent vector $u^{*}$ at a point $R \in \mathcal{S}(\sigma)$ as $t \rightarrow 1$. Let $V_{t}, V^{*}$ be the Zygmund vector fields corresponding to the covering maps $\pi_{t}, \pi: \mathbf{H} \rightarrow R_{t}, R^{*}$ normalized as above. This implies that $V_{t}$ converges pointwise to $V^{*}$ on $\mathbf{R}$ as $t \rightarrow 1$ and the supremum of the Zygmund norms of $V_{t}$ is finite. Then $H\left(V_{t}\right)(x) \rightarrow H(V)(x)$ as $t \rightarrow 1$ uniformly on compact subsets of $\mathbf{R}$ by Lemma 5.1

Let $\dot{\tilde{s}}_{t}, \dot{\tilde{s}}^{*}$ be the infinitesimal shear functions corresponding to the tangent vectors $u_{t}, u^{*}$. Then by Proposition 10.2 we have that

$$
\lim _{t \rightarrow 0} \sum_{j=1}^{l} k_{i, j} H\left(\dot{s}_{t}\right)\left(h_{i, j}\right)=\dot{s}^{*}\left(g_{i}\right) .
$$

This combines with the results of Roger 27. to imply a topological version of a theorem of Masur:

Theorem 10.3. 18] The Weil-Petersson metric on $T(S)$ extends by continuity to the closure $\bar{T}(S)$.

\section{REFERENCES}

[1] W. Abikoff, Degenerating families of Riemann surfaces, Ann. of Math. (2) 105 (1977), no. $1,2944$.

[2] W. Abikoff, C. Earle and S. Mitra, Barycentric extensions of monotone maps of the circle, In the tradition of Ahlfors and Bers, III, 1-20, Contemp. Math., 355, Amer. Math. Soc., Providence, RI, 2004.

[3] L. V. Ahlfors, Lectures on Quasiconformal Mappings, D. Van Nostrand Company, Inc., Princeton, New Jersey, 1966.

[4] L. V. Ahlfors, Some remarks on Teichmüller's space of Riemann surfaces, Ann. of Math. (2) 741961171191.

[5] L. Bers, Spaces of degenerating Riemann surfaces, Discontinuous groups and Riemann surfaces (Proc. Conf., Univ. Maryland, College Park, Md., 1973), pp. 4355. Ann. of Math. Studies, No. 79, Princeton Univ. Press, Princeton, N.J., 1974.

[6] L. Bers, Universal Teichmüller Space, Analytic methods in mathematical physics (Sympos., Indiana Univ., Bloomington, Ind., 1968), pp. 65-83. Gordon and Breach, New York, 1970 .

[7] F. Bonahon, Shearing hyperbolic surface, bending pleated surface and Thurston's sympletic form, Ann. Fac. Sci. Toulouse Math. (6) 5 (1996), 233-297.

[8] F. Bonahon and Y. Sözen, The Weil-Petersson and Thurston symplectic forms, Duke Math. J. 108 (2001), no. 3, 581597.

[9] A. Douady and C. J. Earle, Conformally natural extension of homeomorphisms of the circle, Acta Math. 157 (1986), no. 1-2, 23-48. 
[10] F. Gardiner, Infinitesimal bending and twisting in one-dimensional dynamics, Trans. Amer. Math. Soc. 347 (1995), no. 3, 915937.

[11] F. Gardiner and N. Lakic, Quasiconformal Teichmüller Theory, Mathematical Surveys and Monographs, Volume 76, A.M.S. 2000.

[12] J. Hu, Norms on earthquake measures and Zygmund functions, Proc. Amer. Math. Soc. 133 (2005), no. 1, 193202

[13] J. Kahn and V. Markovic, Random ideal triangulations and the Weil-Petersson distance between finite degree covers of punctured Riemann surfaces, preprint, available on Arxiv.

[14] Y. Katznelson, An introduction to harmonic analysis, Third edition. Cambridge Mathematical Library. Cambridge University Press, Cambridge, 2004.

[15] F. Gardiner and D. Sullivan, Symmetric structures on a closed curve, Amer. J. Math. 114 (1992), no. 4, 683-736.

[16] E. Ghys, Groups acting on the circle, Enseign. Math. (2) 47 (2001), no. 3-4, 329-407.

[17] V. Markovic, Quasisymmetric groups, Journal Amer. Math. Soc. 19 (2006), no. 3, 673-715.

[18] H. Masur, Extension of the Weil-Petersson metric to the boundary of Teichmüller space, Duke Math. J. 43 (1976), no. 3, 623635.

[19] S. Nag and D. Sullivan, Teichmüller theory and the universal period mappings via quantum calculus and the $H^{1 / 2}$ space of the circle, Osaka J. Math. 32 (1995), 1-34.

[20] S. Nag and A. Verjovsky, Diff $\left(S^{1}\right)$ and the Teichmüller spaces, Comm. Math. Phys. 130 (1990), no. 1, 123138.

[21] R. C. Penner, Universal constructions in Teichmüller theory, Adv. Math 98 (1993), 143215.

[22] R. C. Penner, The decorated Teichmüller space of punctured surfaces, Comm. Math. Phys. 113 (1987), 299-339.

[23] R. C. Penner, The Weil-Petersson volume, J. Differential Geom. 35 (1992), no. 3, 559608.

[24] R. C. Penner, On Hilbert, Fourier, and wavelet transforms, Comm. Pure Appl. Math. 55 (2002), no. 6, 772814 .

[25] H. Riemann, Ordinary differential equations and quasiconformal mappings, Invent. Math. 33 (1976), no. 3, 247-270.

[26] G. Riera, A formula for the Weil-Petersson product of quadratic differentials, J. Anal. Math. 95 (2005), 105120.

[27] J. Roger, Factorization rules in quantum Teichmüller theory, preprint, available on Arxiv.

[28] D. Šarić, Real and complex earthquakes, Trans. Amer. Math. Soc. 358 (2006), no. 1, 233249.

[29] D. Šarić, Circle homeomorphisms and shears, Geom. Topol. 14 (2010) 24052430.

[30] W. Thurston, Three-dimensional geometry and topology, Vol. 1. Edited by Silvio Levy. Princeton Mathematical Series, 35. Princeton University Press, Princeton, NJ, 1997.

[31] Minimal stretch maps between hyperbolic surfaces, Unpublished preprint, 1986. arXiv:math.GT/9801039

[32] A. Witten, Coadjoint orbits of the Virasoro group, Comm. Math. Phys. Volume 114, Number 1 (1988), 1-53.

[33] S. Wolpert, Chern forms and the Riemann tensor for the moduli space of curves, Invent. Math. 85 (1986), no. 1, 119145.

[34] S. Wolpert, The Weil-Petersson metric geometry, Handbook of Teichmüller theory. Vol. II, 4764, IRMA Lect. Math. Theor. Phys., 13, Eur. Math. Soc., Zrich, 2009.

[35] S. Wolpert, Behavior of geodesic-length functions on Teichmüller space, J. Differential Geom. 79 (2008), no. 2, 277334.

[36] A. Zygmund, Smooth functions, Duke Math. J. 12, (1945). 4776.

Department of Mathematics, CUNy Queens College, 65-30 Kissena Blvd., Flushing, NY 11367

E-mail address: Dragomir.Saric@qc.cuny.edu

Mathematics PhD program, CUny Graduate Center, 365 Fifth Avenue, New York, NY 10016-4309 